\title{
Simple solutions for downslope pipeline walking on elastic-perfectly-plastic soils
}

\author{
Adriano Castelo \\ PhD Candidate, The University of Western Australia \\ UWA (M053), 35 Stirling Highway CRAWLEY WA 6009, Australia \\ 21445298@student.uwa.edu.au \\ ASME Student Member

\section{David White} \\ Professor, The University of Western Australia \\ UWA (M053), 35 Stirling Highway CRAWLEY WA 6009, Australia \\ david.white@uwa.edu.au

\section{Yinghui Tian} \\ Professor, The University of Western Australia \\ UWA (M053), 35 Stirling Highway CRAWLEY WA 6009, Australia \\ yinghui.tian@uwa.edu.au
}

\begin{abstract}
Pipeline Walking is a phenomenon that occurs when High Pressure and High Temperature pipelines experience axial instability over their operational lifetime, and migrate globally in one direction. Existing analytical solutions treat the axial soil response as rigid-plastic but this does not match the response observed in physical model tests. In this paper, the authors develop a new analytical strategy using elasticperfectly-plastic axial pipe-soil interaction, which leads to more realistic walking rate predictions. The new analytical methodology is benchmarked with a series of Finite Element Analyses (FEA), which constitutes a parametric study performed to test the proposed expressions and improve on the understanding of the influence of axial mobilisation distance.
\end{abstract}

\section{KEYWORDS}

axial resistance; pipe-soil interaction; pipeline walking; finite-element modelling; offshore engineering 


\section{INTRODUCTION}

Offshore pipelines are becoming increasingly important as hydrocarbon sources

3 become more difficult to reach. The global stability of these pipelines in response to

4 operational loading is a critical issue for the design of oil and gas projects. Such stability

5 comprises the actions of hydrodynamic loads and the effects of expansion and

6 contraction triggered by the High-Pressure and High-Temperature (HPHT) operational

7 conditions (usually imposed by frontier reservoirs), which both constitute the major

8 focus of geotechnical design for pipelines.

9 The stability of offshore pipelines is also impacted by the slope of the seabed.

10 New hydrocarbon sources are commonly located in regions with noticeable depth

11 variations, in deep water far from shore. These operational conditions are particularly

12 common in the Gulf of Mexico and Northwest Australia, which are currently in

13 operation, and others that are in development, such as the Brazilian Pre-Salt and the

14 Arctic Area.

15 Threats to the integrity of offshore pipelines by the combination of HPHT

16 conditions and a sloping seabed were first observed by [1]. Later, industry-supported

17 research documented many cases of "axial creeping" now known as the "Pipeline

18 Walking", as per [2].

19 Four mechanisms have been found to incite pipeline walking, as per [3]:

20 1. Tension at the end of the flowline;

212 2. Thermal transients along the line;

22 3. Multiphase fluid behaviour during restart operations; 
4. Seabed slopes along the pipeline route.

Each of the four mechanisms creates an asymmetry in the profile of Effective

25 Axial Force (EAF). This asymmetry generally results in pipeline walking, by causing

26 unequal pipeline displacements during cycles of loading and unloading. This paper

27 focuses on the fourth mechanism.

28 When pipelines are subjected to changes in temperature and pressure, pipeline

29 walking can occur. During the Start-Up (SUp) phase, temperature and pressure

30 increments cause the pipelines to expand axially. This expansion is resisted by the pipe-

31 soil interaction forces which results in effective compression of the pipeline. When

32 pipelines are submitted to temperature and pressure reductions in the Shutdown

33 (SDown) phase, effective tension is induced in the pipeline.

34 For "long" pipelines, the effective compression build up occurs along a sufficient

35 length to induce enough mechanical strain to fully compensate for the thermo-

36 mechanical expansion during the hot stages. For "short" pipelines, the compression build

37 up, due to soil resistance, is not sufficient to fully compensate for the expansion.

38 When "short" pipelines are located on a sloping seabed and are not anchored,

39 cycles of expansion and contraction may cause the pipelines to move with geometric

40 asymmetries between the start-up and shutdown phases. The sloping seabed generates

41 a component of weight to act parallel with the seabed in a downslope direction.

42 Even if pipeline walking is not a limit state in itself, it may present several design

43 challenges, which include:

44

- Overstressing of end connections (and in-line connections); 
- Loss of tension in a steel catenary riser;

- Increased loading leading to lateral buckling;

- Route instability (curve pull out);

- Need for anchoring mitigation.

49 Therefore, pipeline walking must be avoided since its consequences may create 50 downtime and environmental risk, as pointed out by [1].

51 It is known that pre-operational phases may influence the soil resistance during

52 the operational lifetime of a pipeline through the pre-operational embedment. As 53 noticed by [4], typical pipeline embedments can increase the soil axial resistance by 10$5420 \%$. This study considers a range of axial resistance so the results cover the range of 55 conditions that could be created by different values of embedment. In practice, the soil 56 resistance may vary during the pipeline life, in which case the walking rate will also vary

57 as a result of this. The authors would like to clarify that the suggested solutions also 58 apply in cases of varying resistance during the field life requiring only an update on the 59 assessments' inputs.

60 In this paper, focusing exclusively on the seabed slope mechanism, the authors 61 develop a new analytical strategy extending the traditional solution, which uses a Rigid62 Plastic (RP) soil idealization, to a new set of formulations accounting for the Elastic63 Perfectly-Plastic (EP) soil behaviour, which is a simple pipe-soil interaction model [5]. A 64 parametric study is developed with the help of a Finite Element Analysis (FEA) set, which 65 will serve as proof for the proposed set of new equations, leading to more realistic 66 walking rate predictions. 
Journal of Ocean Engineering

Different papers have been published on pipeline walking in the last two decades. Nearly all publications found on this topic are very site-specific [6] and [7], with

70 few exceptions providing generalizations and broad guidance on this issue [2], [3] and $71[8]$.

When the downslope mechanism is taken into consideration the effective axial

73 force plot demonstrates the asymmetry, as referred in section 1 and shown in Fig. 1, for

74 three operational loading cycles. This asymmetry, which accounts for the weight

75 component action, controls the offset distance $X_{a b}$, which is the distance between the

76 Virtual Anchor Sections (VAS) as defined by [2]. $X_{a b}$ is also present in the different

77 profiles of Axial Displacement, $\delta_{x}$, as shown by Fig. 2. In Fig. 2, the axial displacements

78 are shown for the same three operational cycles shown in Fig. 1, throughout the entire

79 pipeline length. In addition, Fig. 2 also provides a detailed progression of the VAS

80 transition along the three operational cycles considered. More attention is given to $X_{a b}$

81 in latter part of this paper.

82 So far pipeline walking has been dealt with through a series of equations which

83 account for a rigid-plastic soil response. In this paper, an extended version of the

84 analytical solution is described for elastic-perfectly-plastic soil behaviour.

85 Fig. 3 provides a schematic view of the Force - Displacement curve (FxD) for a

86 given non-linear soil. It also accounts for rigid-plastic resistance behaviour and presents

87 two different elastic-perfectly-plastic approaches - commonly used as ideal

88 representations for the real non-linear soil. While the magnitude of the limiting axial 
89 resistance depends on soil strength, pipe roughness and drainage conditions [9], these

90 effects are beyond the scope of the present study. Instead, the focus of this paper work

91 is the influence of mobilisation distance, $\delta_{m o b}$, on the pipeline walking phenomenon.

92 The pipe-soil interaction varies with many different properties, [10]. Since this

93 paper simplifies the pipe-soil interaction as an elastic-perfectly-plastic [5], it is simpler to

94 treat the mobilisation displacement as an independent parameter, which allows covering

95 the full parameter space for a wider range of soils. The authors acknowledge that

96 different techniques might be used to obtain the pipe-soil interaction model, but these

97 are not part of this paper scope.

98 Two different elastic-perfectly-plastic fits are shown in Fig. 3. One is a "Stiff Fit" in

99 which the mobilisation distance is denoted $\delta_{\text {mobstiff. }}$ The other is a more compliant case,

100 "Soft Fit", in which the mobilisation distance is denoted $\delta_{\text {mobsoft }}$ In this paper, the

101 mobilisation distances differ by a factor of 3.33, and span the typical range of plausible

102 elastic-perfectly-plastic fits. This is a typical uncertainty range for the non-linear

103 response observed in model tests of axial pipe-soil interaction. Typically, $\delta_{\text {mobstiff }}$ and

$104 \delta_{\text {mobsoft }}$ differ by a factor of up to 5, [9].

105 Fig. 3 brings to light two derived parameters that are explored in the finite 106 element analyses parametric study (section 10): Load and Unload-Reload Areas (the 107 shaded areas presented for the Soft Fit only). They represent the area loss between 108 rigid-plastic and elastic-perfectly-plastic resistance approaches in terms of the FxD 109 curves. They are very useful for the "elastic correction" explanation developed later. 
Journal of Ocean Engineering

111 limiting resistance in the opposite direction is $2 \delta_{m o b}$, and the unloading stiffness matches

112 the loading stiffness.

$113 \quad 3$ PROBLEM DEFINITION

114 To illustrate the behaviour involved in downslope pipeline walking, the properties

115 of a typical example are given in Table 1. General properties, such as temperature loads

116 and geometric data are in keeping with the values presented in Table 1, to allow the

117 results to be applied more broadly in the future.

$118 \quad 4 \quad$ RIGID-PLASTIC ANALYTICAL SOLUTIONS

119 The current design practice - in accordance with [8] - involves three different 120 calculation steps to analytically assess pipeline walking rate under the influence of 121 seabed slope.

122 The first calculation step assesses the distance between the VASs, $X_{a b, R P}$, as 123 presented by Fig. 1:

$$
X_{a b, R P}=\frac{L \tan \beta}{\mu}
$$

124 The second calculation step assesses the change in force in the pipeline, $\Delta S_{S, R P}$,

125 between start-up and shutdown phases over the length of the pipeline denoted by $126 X_{a b, R p}:$

$$
\Delta S_{S, R P}=-W L(\mu \cos \beta-|\sin \beta|)
$$


129 walking behaviour. The walking distance per cycle, $W R_{R P}$ can then be determined in the

130 third and last step by combining equations (1) and (2):

$$
W R_{R P}=\frac{[|\Delta P|+W L|\sin \beta|-W L \mu \cos \beta] L \tan \beta}{E A \mu}
$$

However, equation (3) can be entirely rewritten as:

$$
W R_{R P}=\frac{\left(\Delta S_{S, R P}-\Delta P\right) X_{a b, R P}}{E A}
$$

Equation (4) might also be rewritten more fundamentally as:

$$
W R_{R P}=-\frac{1}{E A}\left(\int_{V A S_{S D o w n, R P}}^{V A S_{S U p, R P}}(\Delta P) d x-\int_{V A S_{S D o w n, R P}}^{V A S_{S U p, R P}}\left(\Delta S_{S}\right) d x\right)
$$

135 additional steps in this analysis).

136 The analytical solutions shown above - equations (1), (2), (3), (4) and (5) - have

137 been used to calculate pipeline walking rates based on the rigid-plastic assumption.

138 Table 2 summarizes the analytical results for [8] based on the general pipeline properties 139 given in Table 1.

$140 \quad 5$ FINITE ELEMENT ANALYSES METHODOLOGY

141 The finite element model used for this paper was a simplified model of a straight

142 pipeline laid on a uniformly sloping seabed using the parameters presented in Table 1.

143 The pipeline was represented by 5001 nodes connected by 5000 equal Euler

144 Bernoulli beams (B33 elements in Abaqus) representing the 5000m long pipeline. Each 145 element, therefore, is 1 metre in length. 
Journal of Ocean Engineering

The pipe-soil interaction was modelled as elastic-perfectly-plastic spring-slider

147 elements connected to each pipeline node. The spring-slider elements were developed

148 as User Elements (UELs) described by a subroutine in FORTRAN.

149 Fig. 4 shows an overall sketch of the finite element model. It presents the

150 uniformly sloped pipeline and provides information about the boundary conditions

151 imposed to all nodes, which can only displace along the local longitudinal axis given the

152 UEL reaction.

153 The spring-slider provided a constant stiffness between zero and a certain

154 prescribed displacement (mobilisation distance) and a corresponding force (according to

155 Hooke's law). If the displacement level exceeds the mobilisation distance, the UEL

156 provides zero tangent stiffness and a constant force, as per the plastic plateau. On

157 reversal, the same stiffness is considered, until the resultant force equals the plastic

158 plateau.

159 The UEL behaviour shown in Fig. 3 is presented in terms of the loads normal to 160 the seabed.

161 This paper considers only weight and temperature as the loads acting on the

162 pipeline. Pressure was disregarded since it can be equally represented by an extra 163 temperature load [11].

164 The effect of the uniform slope is considered as an axial or longitudinal load 165 equivalent to the component of the pipeline weight, as given by:

$$
W_{\text {comp }}=W \sin \beta
$$


Journal of Ocean Engineering

The temperature loads were considered by temperature increments applied

167 directly to the pipeline. Operational cycling was performed taking into account the 168 steady operational profile (start-up) and the rest condition (shutdown).

The analyses were performed by:

1. Generating pipeline (nodes and elements) geometry;

2. Applying boundary conditions and UEL properties;

3. Applying gravity to pipeline;

4. Applying temperature increment (start-up temperature);

5. Applying temperature decrement (shutdown temperature);

6. Iterating phases 4 and 5 (9 times);

7. Extracting results from simulations' outputs.

\section{FINITE ELEMENT ANALYSES COMPARISON WITH RIGID-PLASTIC SOLUTION}

Fig. 5 presents the effective axial force responses for the EP Stiff and the Soft fits;

179 while Fig. 6 and Fig. 7 present the $\delta_{x}$ plots for the EP Stiff Fit and the EP Soft Fit, 180 respectively.

181 From the rigid-plastic case [3], the zero displacement point is exactly the same as

182 the maximum effective axial force point (Table 3). However, the elastic-perfectly-plastic

183 FE results show that the point of zero displacement no longer coincides with the point of 184 maximum effective axial force.

185 As defined by [2], the VASs are the sections where the $\delta_{x}$ is zero and for the rigid186 plastic soil response the VAS and the point of highest effective axial force coincide, which 187 makes the solution proposed by [8] perfectly applicable for rigid-plastic soils. 
189 used by [3] and [8]. Thus, $X_{a b}$ needs to be redefined. In addition, the points on the pipe 190 with zero net movement $\left(\delta_{x}=0\right)$ over the period of temperature change (either start-up

191 or shutdown) are not stationary over this period but they move initially in one direction

192 then return to their original position. Here, these sections with zero net movement are 193 called "Stationary Points" (SP). While $\delta_{x}$ during the temperature change phase is ideally

194 zero for these sections, in fact they move through a cycle of displacement and return to

195 the original position at the end of the expansion or contraction. Fig. 8 shows the 196 mentioned behaviour for stationary points during some load phases (for the EP Stiff Fit)

197 along with a schematic plot of the finite element model to clarify the location of these 198 stationary points. It is important to highlight that there will be one stationary point per 199 loading phase, which will remain at the same pipeline Kilometre Post (KP), represented 200 by the model nodes, as long as the conditions (temperature, soil, geometry, etc.) also

201 remain the same during the operational lifetime.

202 In the following analysis, $X_{a b}$ is defined as the distance between the stationary 203 points. This definition is more useful than the distance between the maxima in the

204 effective axial force profiles because the walking rate per cycle is fundamentally related 205 to the integrated change in effective axial force in the length of pipe between the 206 stationary points.

$207 \quad 7 \quad X_{a b}$ FOR ELASTIC-PERFECTLY-PLASTIC SOIL

208 The three different values for $X_{a b}\left(X_{a b, R P}, X_{a b, E P_{-} S t i f f}\right.$ and $\left.X_{a b, E P_{-} S o f t}\right)$ are compared 209 to $\delta_{m o b}$, in Fig. 9, which shows the linear dependence of $X_{a b}$ on $\delta_{m o b}$. Imagining there is a 
210 certain level of mobilisation distance which makes $X_{a b}$ to be equal to zero (and

211 consequently ceases the walking pattern), represented by $\delta_{\text {null, }}$ which will be given later

212 in this paper, the following linear equation might be written:

$$
X_{a b, E P}=X_{a b, R P}\left(1-\frac{\delta_{m o b}}{\delta_{\text {null }}}\right)
$$

This definition of $X_{a b, E P}$ for use in equation (4) is now defined for elastic-perfectly-

214 plastic soils, there is only one other missing $-\Delta S_{S, E P}$ - in order for the elastic-perfectly-

215 plastic walking rate be derived analytically.

\section{$8 \Delta S_{S}$ FOR ELASTIC-PERFECTLY-PLASTIC SOIL}

For rigid-plastic soils, $\Delta S_{S}$ can be obtained directly from the basic problem

218 parameters using equation (2). For elastic-perfectly-plastic soils, however, $\Delta S_{S}$ is not

219 straight forward, as the effective axial force profile is not triangular. For this reason, the

220 effective axial force equations need to be redefined by adopting the solution for an

221 elastic column compressed within an elastic medium, as used in the analysis of piles. This

222 leads to a second order linear differential equation which represents the displacement,

$223 \delta$, along the longitudinal axis, $x$, as shown by equation (8) from [12].

$$
\delta=K_{1} e^{\xi x}+K_{2} e^{-\xi x}
$$

224 where $K_{1}$ and $K_{2}$ are arbitrary constants, and $\xi$ is exponential factor. More detail

225 about these parameters is given in Appendix B.

226 However, before solving the differential equation the boundary conditions

227 among the different behaviour patterns along the pipe route need to be defined.

228 Table 4 presents the physical boundaries that should be considered for the

229 elastic-perfectly-plastic effective axial force calculation, which segregates the different 
230 zones of the pipeline. For pipeline zones $Z 1$ and $Z 4$ effective axial force is equivalent to

231 the rigid-plastic solution with straight line behaviour and constant gradient - equations

232 (9) and (10):

$$
\begin{aligned}
& W(\mu \cos \beta+\sin \beta) \\
& W(\mu \cos \beta-\sin \beta)
\end{aligned}
$$

233 In contrast to zones $Z 1$ and $Z 4$, the behaviour of the $Z 2$ and $Z 3$ central zones (in

234 the vicinity of the highest effective axial force section), creates two different parabolic 235 curves (within the effective axial force plot), whose gradients vary from 0 to the values 236 given by equations (9) and (10).

Fig. 10 presents a schematic plot accounting the physical boundaries and also the

238 revised solution for a hypothetic case.

i $\quad \delta_{x}$ Boundary Conditions

Considering the physical boundaries and their outcomes in terms of

241 displacement, $\delta$, it is clear that displacements at $x_{23}$ are zero, while at $x_{12}$ and $x_{34}$

242 displacements are equal to $\delta_{m o b}$, where the soil resistance is fully mobilised.

243 ii Effective Axial Force Boundary Conditions

244 From Fig. 10 it is clear that some boundary conditions must be respected when 245 obtaining the analytical elastic-perfectly-plastic effective axial force response; which are:

- Continuity of slope for the three zone boundaries;

- Continuity of effective axial force at the three zone boundaries.

249 Table 5. The question mark in Table 5 might only be answered after the differential 250 equation is solved and an expression for the effective axial force calculation is reached. 
Hence, a general equation was written as follows:

$$
\left(\frac{d F}{d x}\right)_{x}=\left\{\begin{array}{cl}
\mu W_{Z 1}, & \delta_{x} \leq-\delta_{m o b} \\
\left(\frac{\mu W_{Z 1}}{\delta_{m o b}}\right) \delta_{x}, & -\delta_{m o b}<\delta_{x}<0 \\
\left(\frac{\mu W_{Z 4}}{\delta_{m o b}}\right) \delta_{x}, & 0<\delta_{x}<\delta_{m o b} \\
\mu W_{Z 4}, & \delta_{x} \geq \delta_{m o b}
\end{array}\right.
$$

253 considered, the weight component acting on the pipe due to the seabed slope.

254 iii Effective Axial Force Pipe Differential Equation

255 Observing the effective axial force boundary conditions and their implications, 256 the effective axial force differential equation could be written as:

$$
\begin{aligned}
& E A F_{(x)}
\end{aligned}
$$

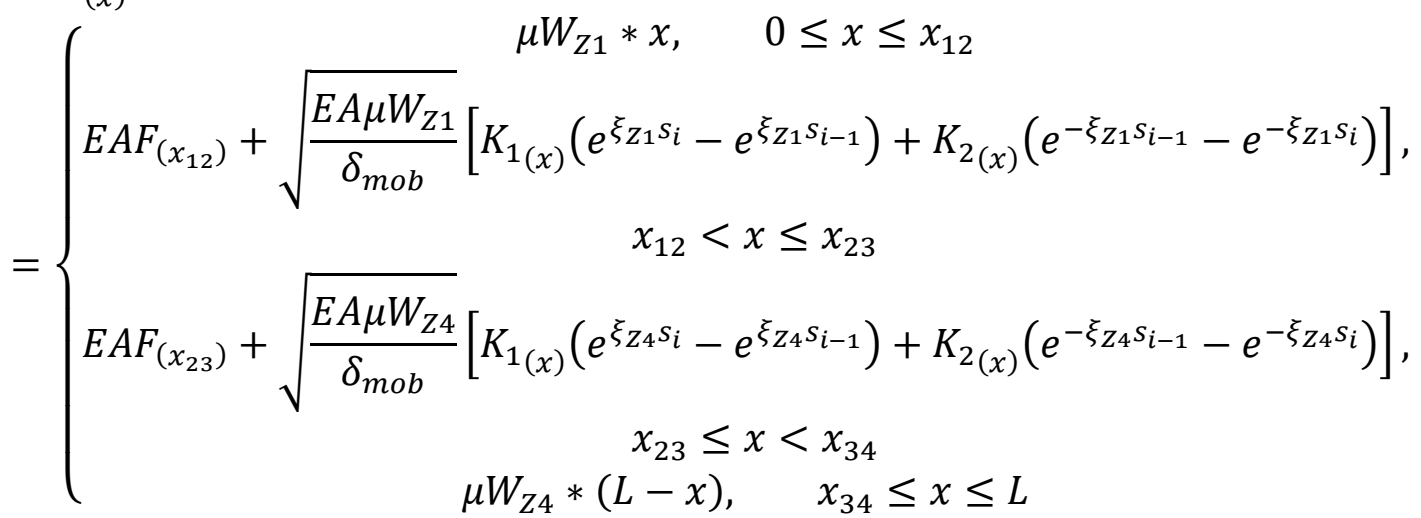

258 towards equation (12), based on the strategy adopted in .

With equation (12) the unknown values in Table 5 are derived and the full

260 effective axial force profiles can be deduced via iteration on the position of $x_{23}$. 
265 (13), and as schematically shown by Fig. 11.

$$
\int_{S P_{S D o w n}}^{S P_{\text {SUp }}}\left(\Delta S_{S}\right) d x=-(\mid \text { Area } 1|+| \text { Area } 2|+| \text { Area } 3|+| \text { Area } 4 \mid)
$$

where each area represents the partial integral of effective axial force in terms of

$267 x$ coordinate accounting the physical boundaries as seen in Fig. 11.

9 WALKING RATE FOR ELASTIC-PERFECTLY-PLASTIC SOIL

270 can be derived. Taking into account equation (5), the general modifications are:

$$
W R_{E P}=-\frac{1}{E A}\left(\int_{S P_{S D o w n, E P}}^{S P_{S U p, E P}}(\Delta P) d x-\int_{S P_{S D o w n, E P}}^{S P_{S U p, E P}}\left(\Delta S_{S}\right) d x\right)
$$

272 study was conducted.

- Pipeline length;

- $\quad$ Pipeline submerged weight;

- Friction factor;

- Route overall slope. 
Journal of Ocean Engineering

Since the focus of this paper is the influence of axial mobilisation distance, eight

282 different values of $\delta_{m o b}$ were considered, in terms of pipeline steel outside diameter

283 (OD), (0.03OD, 0.05OD, 0.06OD, 0.100D, 0.15OD, 0.200D, 0.33OD and 0.50OD), giving a

284 total of 648 cases.

285

All 648 cases were modelled using the same finite element analyses solution. All

286 respected the general behaviour for the pipeline walking phenomenon as expected

287 (including the revised solutions).

Fig. 12 presents the finite element analyses results for $X_{a b E P}$ plotted against $\delta_{m o b}$

289 for the $1^{\circ}$ seabed slope while Fig. 13 compares $X_{a b}$ achieved through finite element

290 analyses and the equations proposed in this paper. Fig. 14, Fig. 15, Fig. 16 and Fig. 17

291 provide the same results for $2^{\circ}$ and $3^{\circ}$ seabed slopes, respectively.

292 In Fig. 13, Fig. 15 and Fig. 17 the results were plotted along with a line 293 representing the equation (7) for each case. The finite element analyses results clearly 294 validate equation (7).

295 At this stage, the results obtained for $X_{a b}$ using the suggested formulation 296 (equation (7)) and the finite element analyses' results were statistically analysed. For the

$2971^{\circ}$ slope, the coefficient of determination, $R^{2}$, is equal to 0.986 ; whilst for $2^{\circ}$ and $3^{\circ}, R^{2}$ is

298 equal to 0.997 and 0.998 , respectively. It is clear that the proposed methodology has a 299 very strong accuracy. The authors also looked into the reason for the difference noticed 300 in the $1^{\circ}$ models, and it was found that some finite element models had an accidental 301 limitation in terms of mesh. This generated a numerical noise that was reflected in the 
302 overall results. The noise can be eliminated through the use of a finer mesh in the 303 models, thus retaining their applicability to any slope.

$304 \quad$ Fig. 18 shows the finite element model results for $W R_{E P}$ plotted against $\delta_{\text {mob }}$ for

305 the $1^{\circ}$ seabed slope. Fig. 20 and Fig. 22 give the same results for $2^{\circ}$ and $3^{\circ}$ seabed slopes.

306 Fig. 19, Fig. 21 and Fig. 23 present the comparison between finite element analyses and 307 equation results.

308 Again, applying some statistics to the results shown by Fig. 19, Fig. 21 and Fig. 23,

309 the coefficient of determination, $R^{2}$, was calculated to be 0.985 (for $1^{\circ}$ slope), 0.997 (for $3102^{\circ}$ slope) and 0.999 (for $3^{\circ}$ slope). These results confirm the level of accuracy of the

311 findings of this paper and reinforce the applicability of the proposed methodology.

312 As it can be seen, the analytical expressions shown in sections 7, 8 and 9 agree

313 closely with the finite element analyses results, as shown by the plots from Fig. 12 to Fig.

31423.

315 Hence, for any straight pipeline resting on any sloping seabed with an elastic-

316 perfectly-plastic soil we can conclude that the realistic walking rate might be written as:

$$
W R_{E P}=W R_{R P}-\text { ElasticCorr }
$$

Where the elastic correction, ElasticCorr, is equivalent to:

$$
\text { ElasticCorr }=2\left(\frac{\text { Unload }- \text { Reload Area }}{\Delta F}\right)
$$

319 the single-spring elastic-perfectly-plastic approach, the entity Elastic Correction equals:

$$
\text { ElasticCorr }=2\left(\frac{2 \mu W \cos \beta \delta_{m o b}}{2 \mu W \cos \beta}\right)=2 \delta_{m o b}
$$


321 to be:

$$
\delta_{\text {null }}=\frac{W R_{R P}}{2}
$$

\section{CONCLUSIONS \& FINAL REMARKS}

This paper provides an analytical solution that solves pipeline walking problems

324 for elastic-perfectly plastic (EP) pipe-soil response, benchmarked and validated against

325 finite element analyses performed with an elastic-perfectly-plastic user-defined element.

326 These revised solutions improve understanding of the parameters involved in elastic-

327 perfectly-plastic soil behaviour for pipeline walking assessment. The paper resolves how

328 the fundamental solution for rigid-plastic pipe-soil interaction requires expansion to

329 allow for elasticity. It is shown that the "Stationary Points", which have zero movement

330 during changes in the pipe temperature, do not coincide with the positions of maximum

331 effective axial force (EAF). This is an important distinction compared to the rigid-plastic

332 solution, in which the term "Virtual Anchor Point" is well-established as both the

333 Stationary Point and the position of maximum effective axial force. Using the revised

334 Stationary Points, the resulting mathematical proof shows the swept area within the

335 effective axial force plot during a change in temperature remains a valid method to

336 assess the pipeline expansion and contraction and therefore the pipeline walking.

337 Relative to the rigid-plastic solution, the correction for elasticity is equivalent to the loss

338 in area represented by the Unload-Reload Area inherent to the FxD soil curve.

339 Common solutions for pipeline walking, in which the soil is treated as rigid-

340 plastic, invariably derive overestimates of walking action. Besides being unrealistic, a 
341 magnified walking rate can be onerous for projects, leading to additional effort and cost

342 to mitigate pipeline walking.

343 Therefore, it is important to identify and apply realistic soil properties, and the

344 solution in this paper allows the elastic-perfectly-plastic rather than rigid-plastic

345 approach to be used.

346 The walking mechanism, explored in this paper, can now be assessed by a set of

347 analytical expressions for walking evaluation, based on the general problem properties,

348 such as, overall route slope, temperature variation and pipeline geometric data. These

349 expressions were validated against a finite element analyses set.

\section{ACKNOWLEDGMENT}

351 This research forms part of the activities of the Centre for Offshore Foundation

352 Systems (COFS), established under the Australian Research Council's Research Centres

353 Program and currently supported as a node of the Australian Research Council Centre of

354 Excellence for Geotechnical Science and Engineering. The authors acknowledge the 355 support from The University of Western Australia and Shell. 
Journal of Ocean Engineering

\section{NOMENCLATURE}

358

Steel Young's Modulus

$E P \quad$ elastic-perfectly-plastic

EAF effective axial force

FEA finite element analysis

FEM finite element model

FxD force $x$ displacement curve

HP high pressure

$H T \quad$ high temperature

KP kilometre post

$K_{1}$ differential equation constant 1

$K_{2}$ differential equation constant 2

$L \quad$ pipeline length

$O D \quad$ overall pipe outside diameter

$R P \quad$ rigid-plastic

$R^{2} \quad$ coefficient of determination

$s \quad$ distance to stationary point 


\begin{tabular}{|c|c|}
\hline SDown & shutdown phase \\
\hline SUp & start-up phase \\
\hline$S P$ & stationary point \\
\hline$t$ & steel wall thickness \\
\hline UEL & user element \\
\hline VAS & virtual anchor section \\
\hline W & pipeline submerged weight \\
\hline$W_{\text {comp }}$ & pipeline weight component \\
\hline$W R$ & walking rate \\
\hline$x$ & axial coordinate along pipe length \\
\hline$x_{12}$ & physical boundary between $Z 1$ and $Z 2$ \\
\hline$x_{23}$ & physical boundary between $Z 2$ and $Z 3$ \\
\hline$x_{34}$ & physical boundary between $Z 3$ and $Z 4$ \\
\hline$x_{a b}$ & distance between stationary points \\
\hline$Z 1$ & route's zone 1 \\
\hline$Z 2$ & route's zone 2 \\
\hline$Z 3$ & route's zone 3 \\
\hline$Z 4$ & route's zone 4 \\
\hline$\alpha$ & steel thermal expansion coefficient \\
\hline
\end{tabular}


Journal of Ocean Engineering

b

$\Delta S_{S} \quad$ effective axial force variation over Xab

$\Delta P \quad$ change in fully constrained force

$\Delta T$

$\delta$

$\delta_{m o b}$

$\delta_{\text {null }}$

$\delta_{x}$

$\varepsilon_{\text {Mech }}$

$\varepsilon_{\text {Thermal }}$

$\varepsilon_{\text {Total }}$

$\mu$

$\mu W_{Z 1}$

$\mu W_{z 4}$

$v$

$\xi$

seabed slope angle

temperature variation

general displacement

mobilisation distance

non-walking mobilisation distance

axial displacement

mechanical strain

thermal strain

total strain

axial friction coefficient

resistant friction component in zone 1

resistant friction component in zone 4

steel Poisson coefficient

differential equation exponential factor $\xi$

359

360 
Journal of Ocean Engineering

361

362

363

364

365

366

367

368

369

370

371

372

373

374

375

376

377

378

379

380

381

382

383

384

385

386

387

388

389

390

391

392

393

394

395

396

397

398

399

400

401

402

\section{REFERENCES}

[1] K. Tornes, B. A. Ose, J. Jury, and P. Thomson, "Axial Creeping of High Temperature Flowlines Caused by Soil Ratcheting," in Proceedings of the ETCE/OMAE2000 Joint Conference, 2000.

[2] M. Carr, D. Bruton, and D. Leslie, "Lateral Buckling and Pipeline Walking, a Challenge for Hot Pipelines," in Proceedings of the Offshore Pipeline Technology Conference 2003, 2003.

[3] D. Bruton, F. Sinclair, and M. Carr, "Lessons Learned From Observing Walking of Pipelines with Lateral Buckles, Including New Driving Mechanism and Updated Analysis Models," in Proceedings of the Offshore Technology Conference 2010, 2010.

[4] D. White, M. E. Campbell, N. P. Boylan, and M. F. Bransby, "A new framework for axial pipe-soil interaction illustrated by shear box tests on carbonate soils," in Proceedings of the 2012 International Conference on Offshore Site Investigation and Geotechnics, 2012.

[5] D. Bruton, D. J. White, M. Carr, and J. Cheuk, "Pipe-Soil Interaction During Lateral Buckling and Pipeline Walking - The SAFEBUCK JIP," in Proceedings of the Offshore Technology Conference 2008, 2008.

[6] D. Carneiro and A. Castelo, "Walking Analyses of HP/HT Pipelines with Sliding End Structures Using Different FE Models," in Proceedings of the Rio Pipeline Conference \& Exposition 2011, 2011.

[7] D. Jayson, P. Delaporte, J.-P. Albert, M.-E. Prevost, D. Bruton, and F. Sinclair, "Greater Plutonio Project - Subsea Flowline Design and Performance," in Proceedings of the Offshore Flowline Technology Conference 2008, 2008.

[8] M. Carr, F. Sinclair, and D. Bruton, "Pipeline Walking - Uderstanding the Field Layout Challenges, and Analytical Solutions Developed for the SAFEBUCK JIP," in Proceedings of the Offshore Technology Conference 2006, 2006.

[9] D. J. White, S. Ganesan, M. Bolton, D. Bruton, J.-C. Ballard, and T. Langford, "SAFEBUCK JIP - Observations of Axial Pipe-Soil Interaction from Testing on Soft Natural Clays," in Proceedings of the Offshore Technology Conference 2011, 2011.

[10] A. J. Hill, D. White, D. Bruton, T. Langford, V. Meyer, R. Jewell, and J.-C. Ballard, "A New Framework for Axial Pipe-Soil Resistance, Illustrated by a Range of Marine Clay Datasets," in Proceedings of the 2012 International Conference on Offshore Site Investigation and Geotechniques, 2012.

[11] R. E. Hobbs, "In-Service Buckling of Heated Pipelines," ASCE Journal of Transportation Engineering, vol. 110, pp. 175-189, 1984.

[12] M. Randolph, "A Theoretical Study of the Performance of Piles," PhD Thesis, University of Cambridge, 1977. 


\section{Figure Captions List}

Fig. 1 EAF diagrams for SUp and SDown

Fig. $2 \quad \delta_{x}$ diagrams for SUp and SDown phases

Fig. 3 RP \& EP soil responses

Fig. $4 \quad$ Finite element model sketch

Fig. 5 EAF plot (Zoom)

Fig. $6 \quad \delta_{x}$ plot for Stiff Fit (Zoom)

Fig. $7 \quad \delta_{x}$ plot for Soft Fit (Zoom)

Fig. $8 \quad x$ Coordinate for the SPs

Fig. $9 \quad X_{a b E P}$ results against $\delta_{m o b}$

Fig. 10 Schematic plot accounting physical boundaries

Fig. 11 Schematic EAF plot with the partial areas highlight

Fig. $12 \quad X_{a b E P}$ results for $1^{\circ}$ slope

Fig. $13 X_{a b E P}$ results - Numerical (FEA) \& Calculated (Equations) - for $1^{\circ}$ slope

Fig. $14 \quad X_{a b E P}$ results for $2^{\circ}$ slope

Fig. $15 X_{a b E P}$ results - Numerical (FEA) \& Calculated (Equations) - for $2^{\circ}$ slope

Fig. $16 \quad X_{a b E P}$ results for $3^{\circ}$ slope

Fig. $17 X_{a b E P}$ results - Numerical (FEA) \& Calculated (Equations) - for $3^{\circ}$ slope

Fig. $18 W R_{E P}$ results for $1^{\circ}$ slope 
Journal of Ocean Engineering

Fig. $19 W R_{E P}$ results - Numerical (FEA) \& Calculated (Equations) - for $1^{\circ}$ slope

Fig. $20 \quad W R_{E P}$ results for $2^{\circ}$ slope

Fig. $21 W R_{E P}$ results - Numerical (FEA) \& Calculated (Equations) - for $2^{\circ}$ slope

Fig. $22 \quad W R_{E P}$ results for $3^{\circ}$ slope

Fig. $23 W R_{E P}$ results - Numerical (FEA) \& Calculated (Equations) - for $3^{\circ}$ slope 
Journal of Ocean Engineering

Table $1 \quad$ Preliminary example properties

Table $2 \quad$ RP analytical results

Table 3 EP FEA results

Table $4 \quad$ Pipeline zoning

Table $5 \quad$ EAF boundary conditions

Table 6 FEA parametric variables 
410 Fig. 1 EAF diagrams for SUp and SDown phases

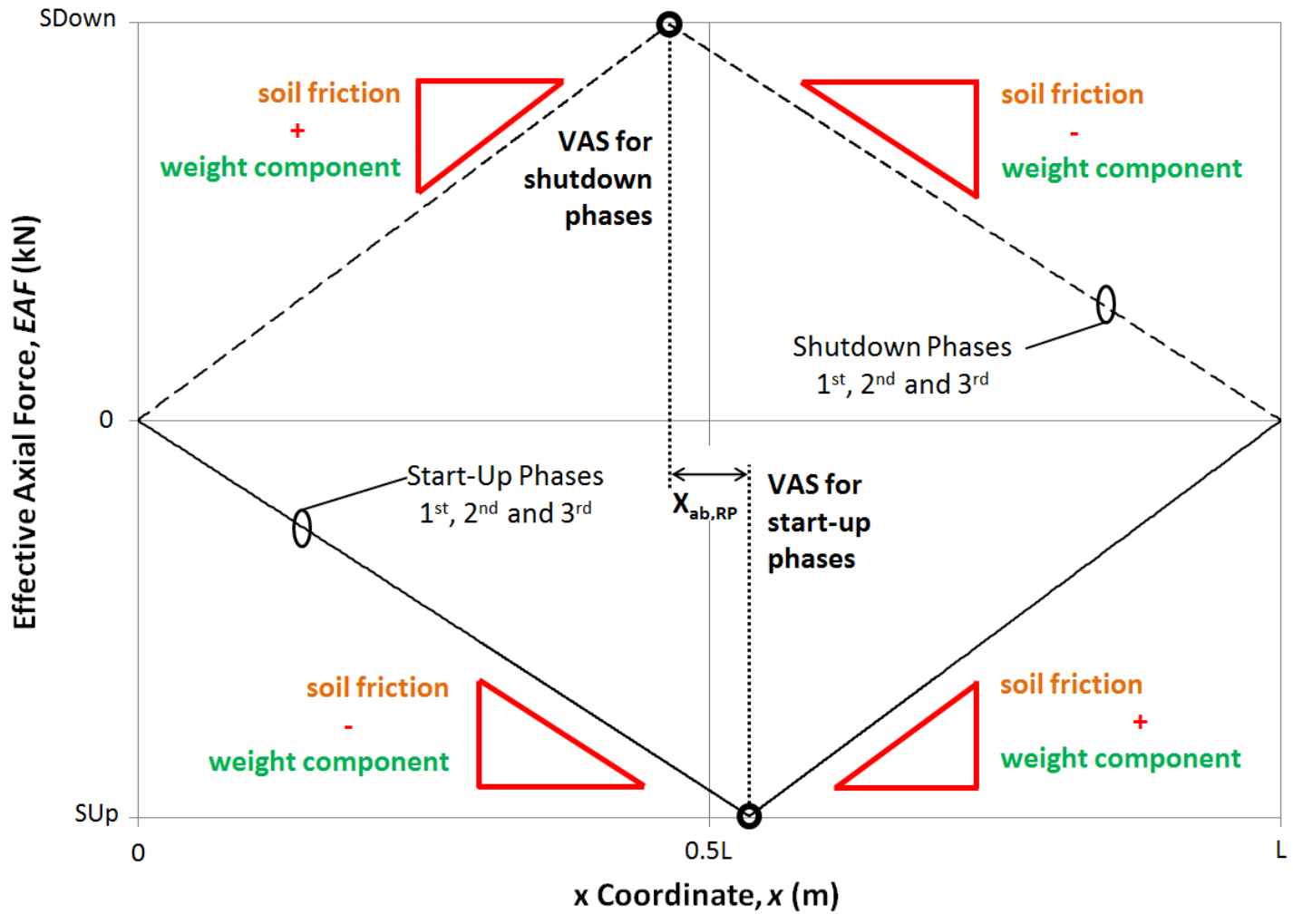

411

412

413 
Journal of Ocean Engineering

\section{Fig. $2 \delta_{x}$ diagrams for SUp and SDown phases}

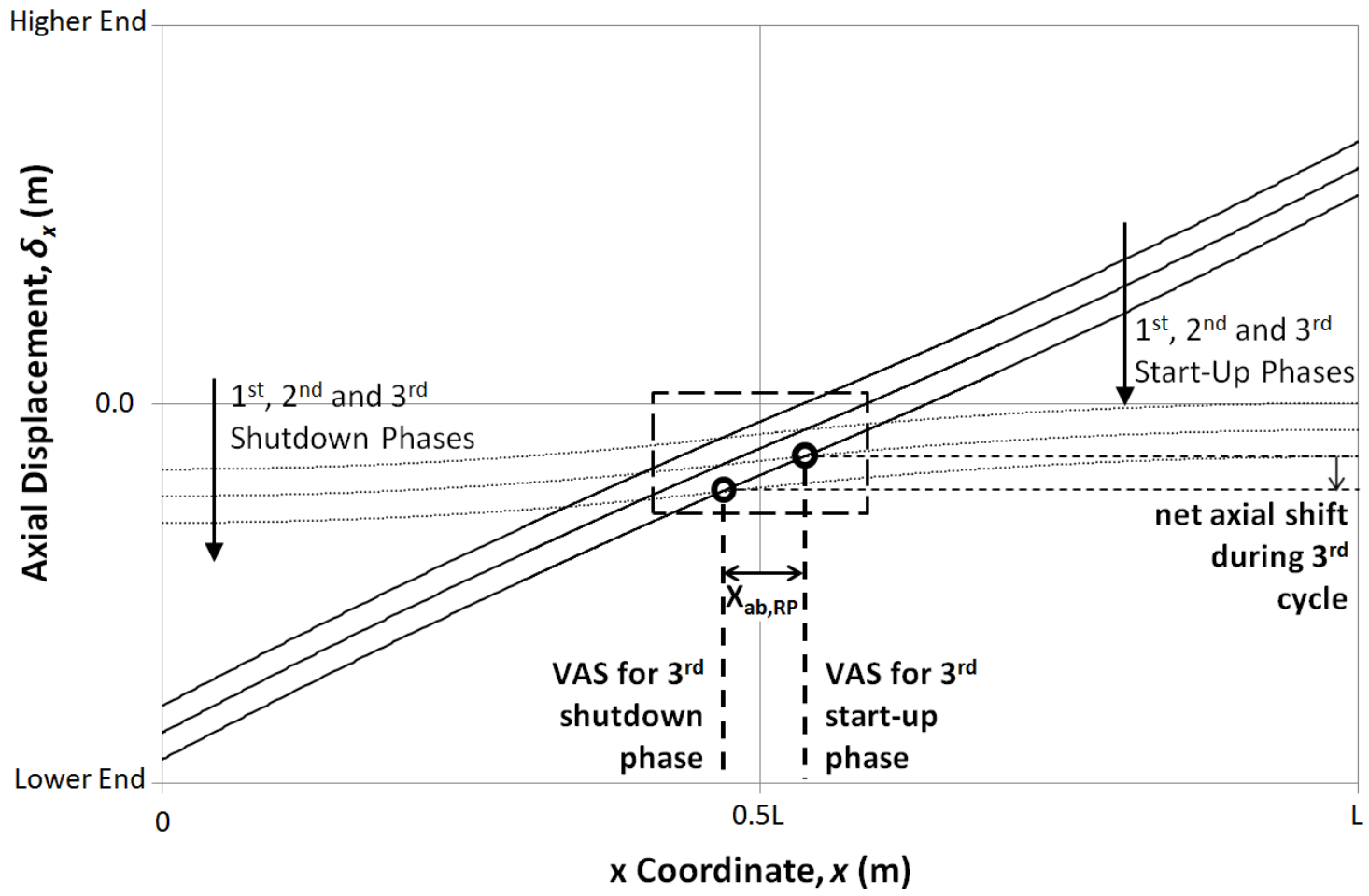

415

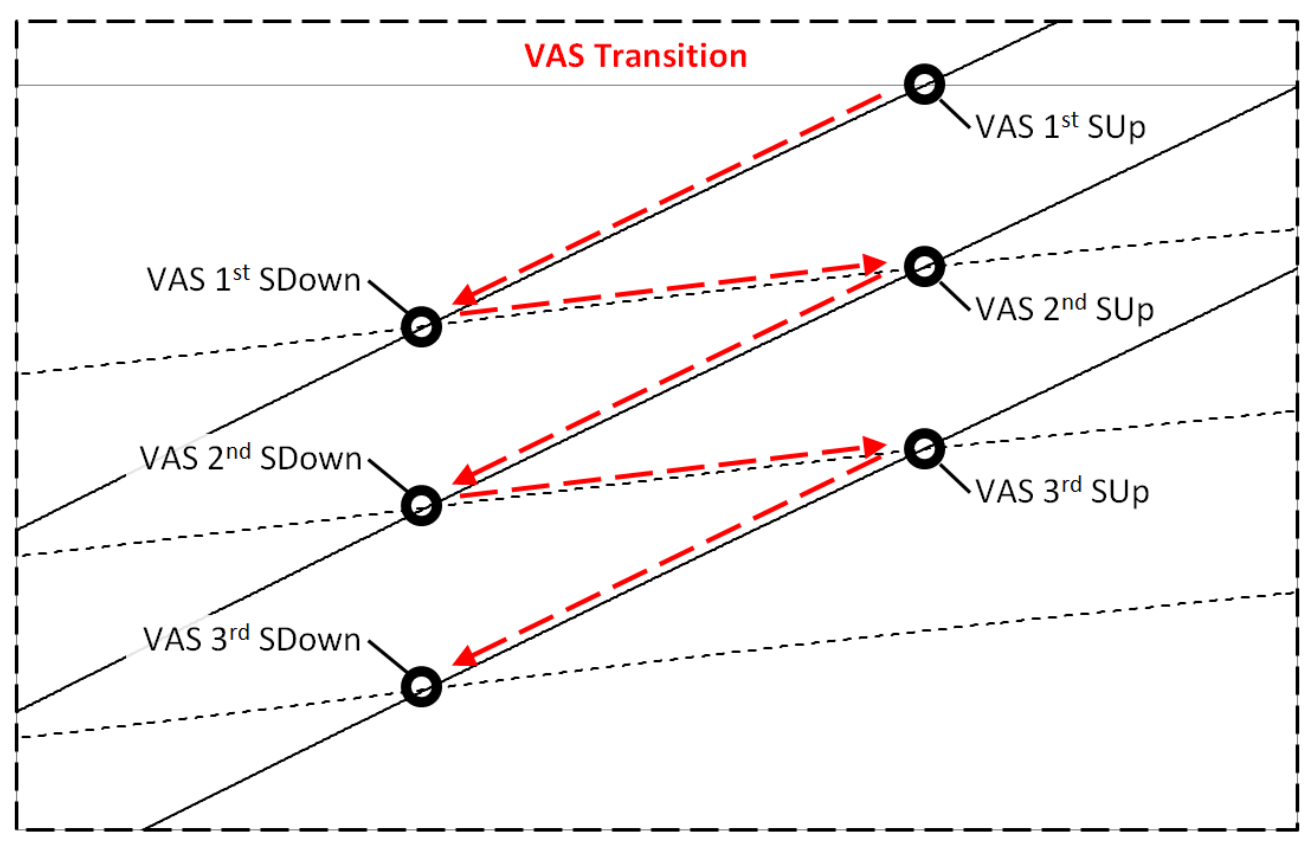


Journal of Ocean Engineering

419

Fig. 3 RP \& EP soil responses

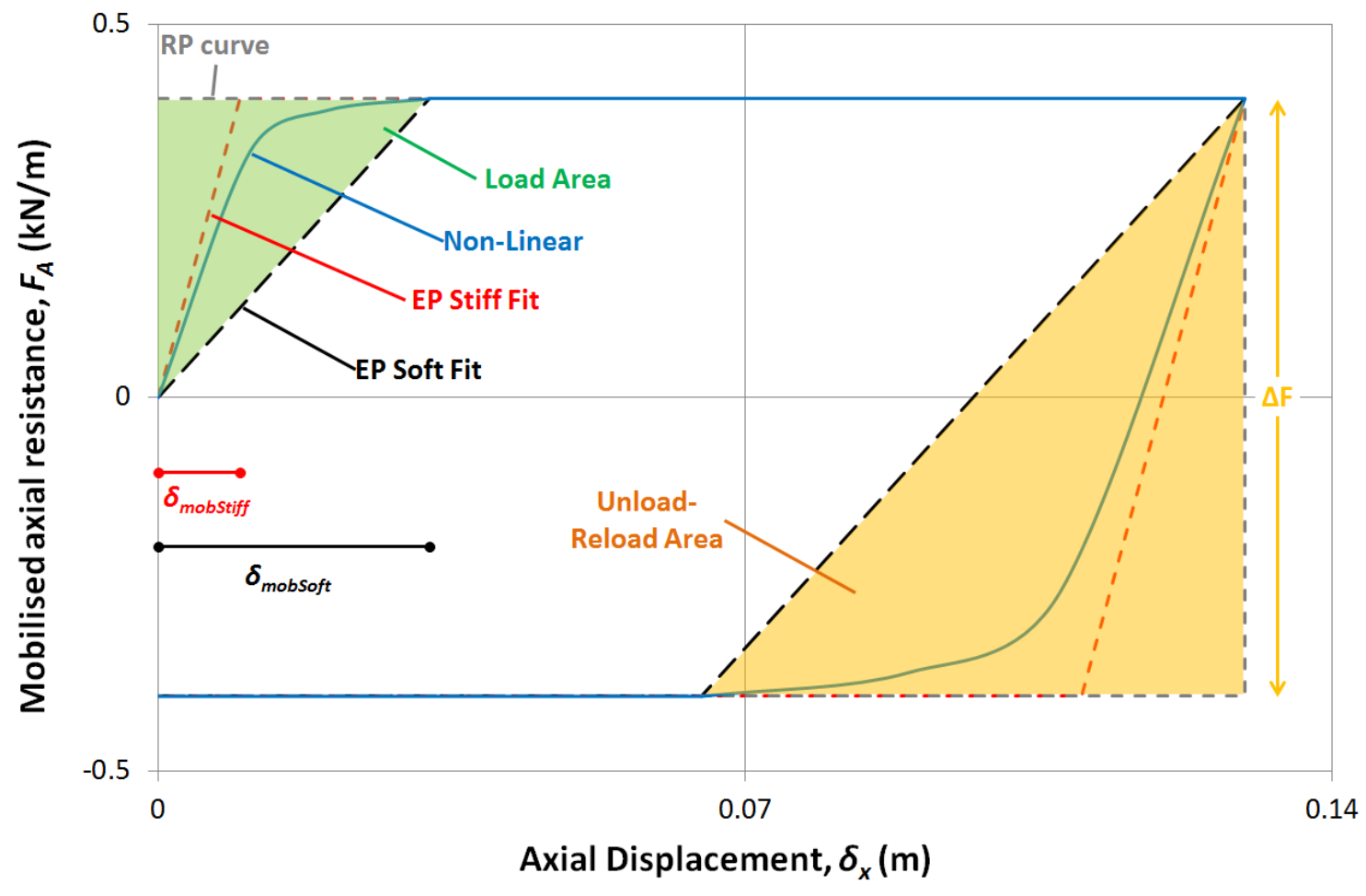

421

422 
Journal of Ocean Engineering

423 Fig. 4 Finite element model sketch

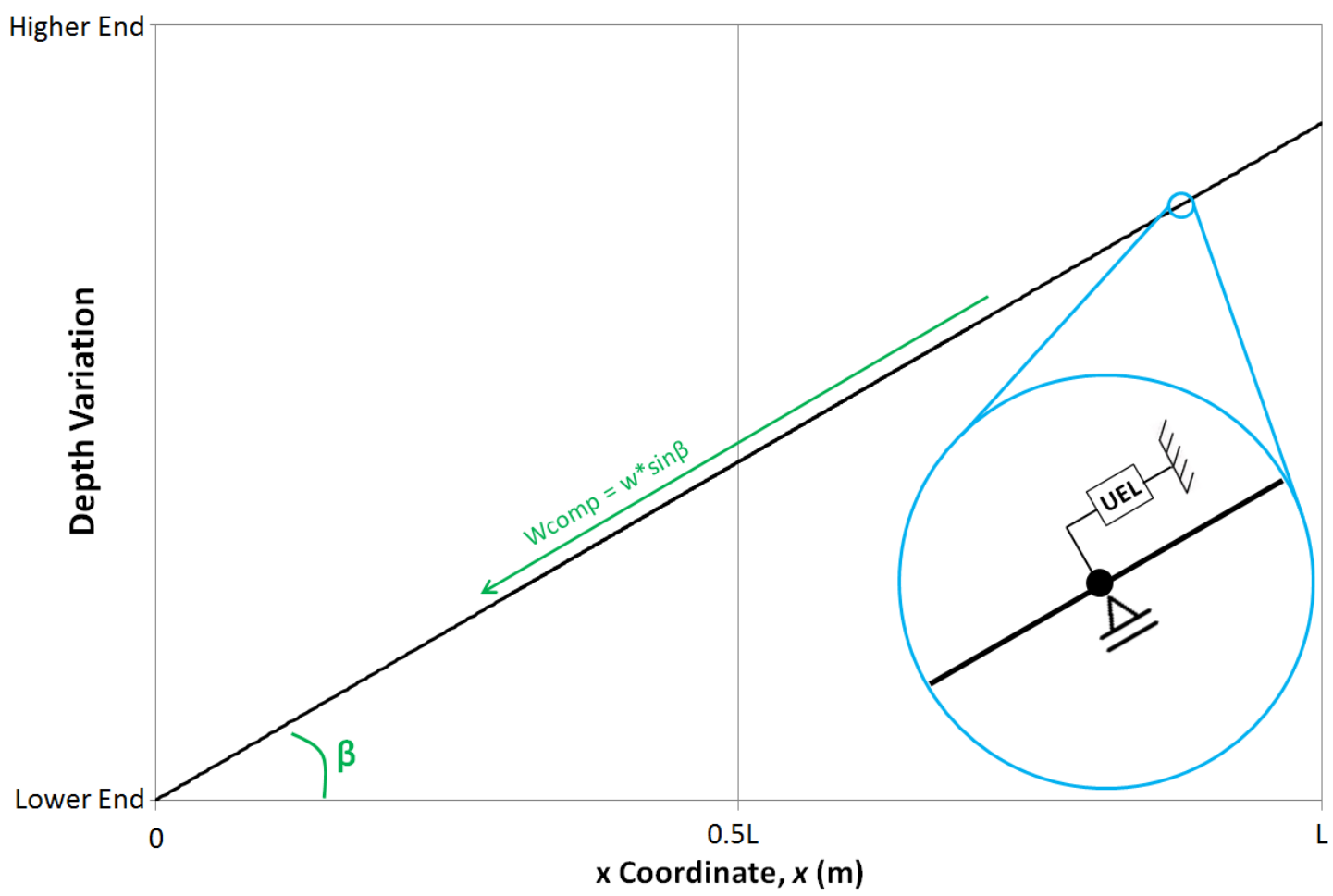

424

425 
Fig. 5 EAF plot (Zoom)

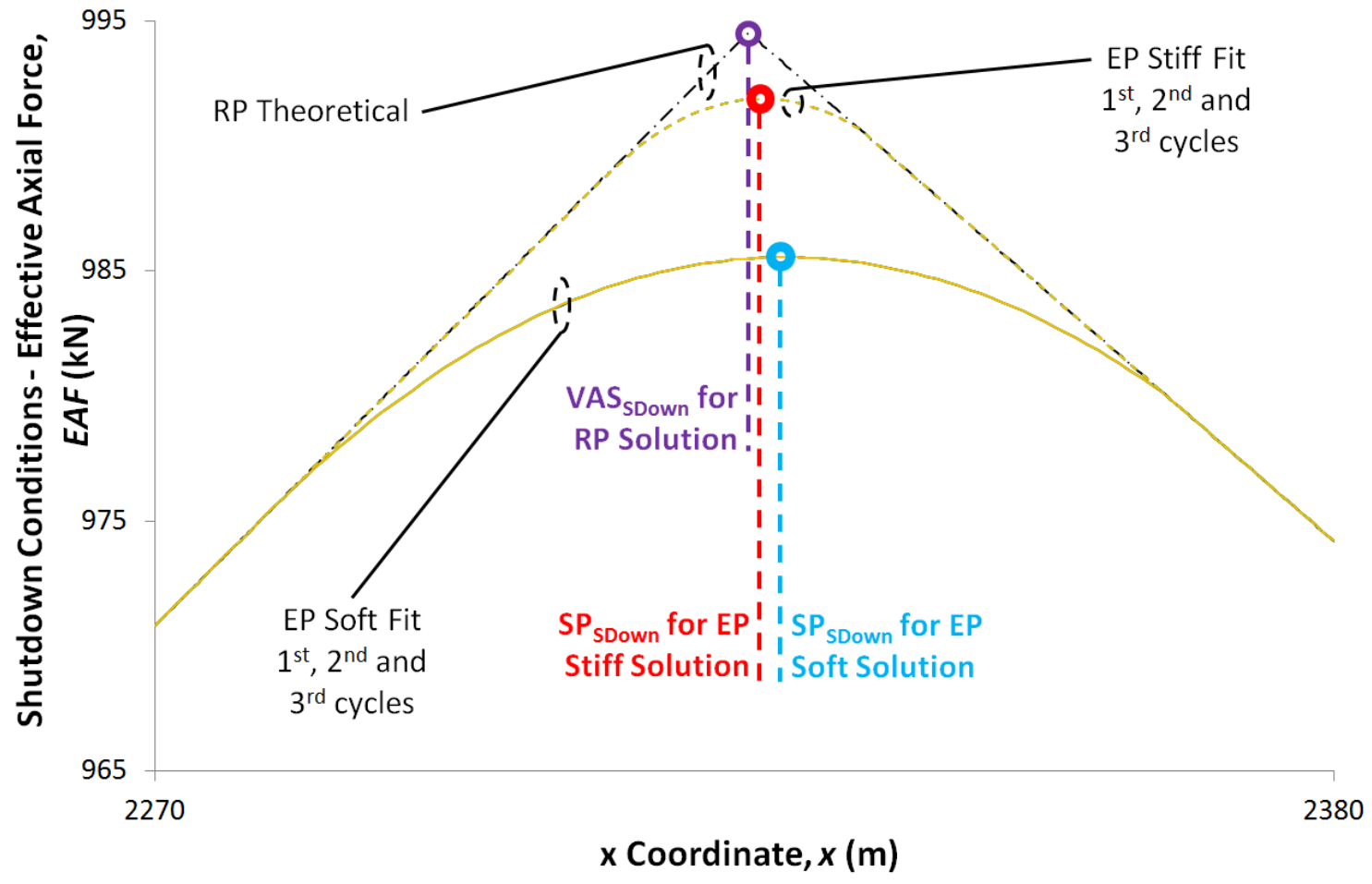

427

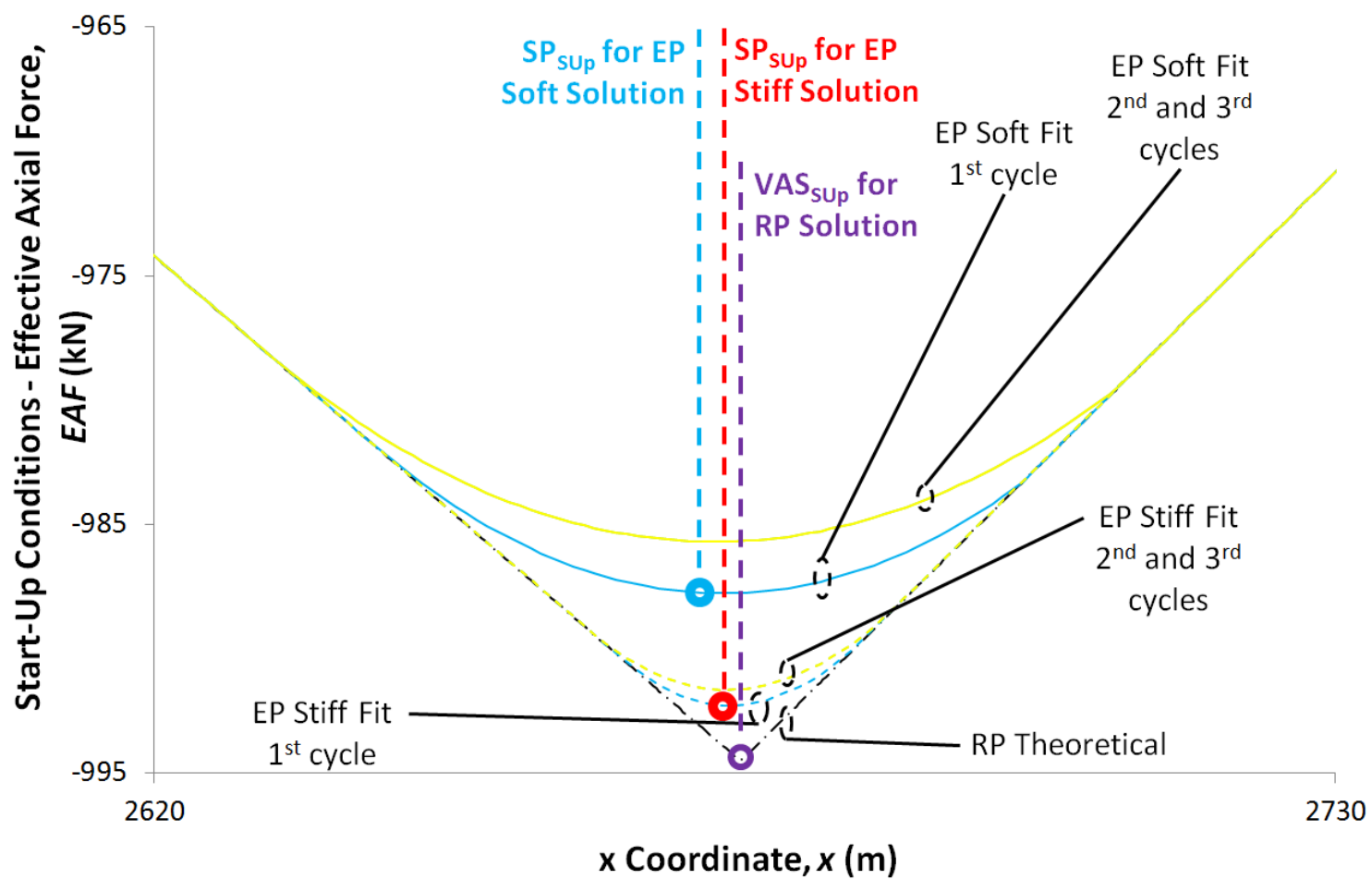




\section{Fig. $6 \boldsymbol{\delta}_{\boldsymbol{x}}$ plot for Stiff Fit (Zoom)}

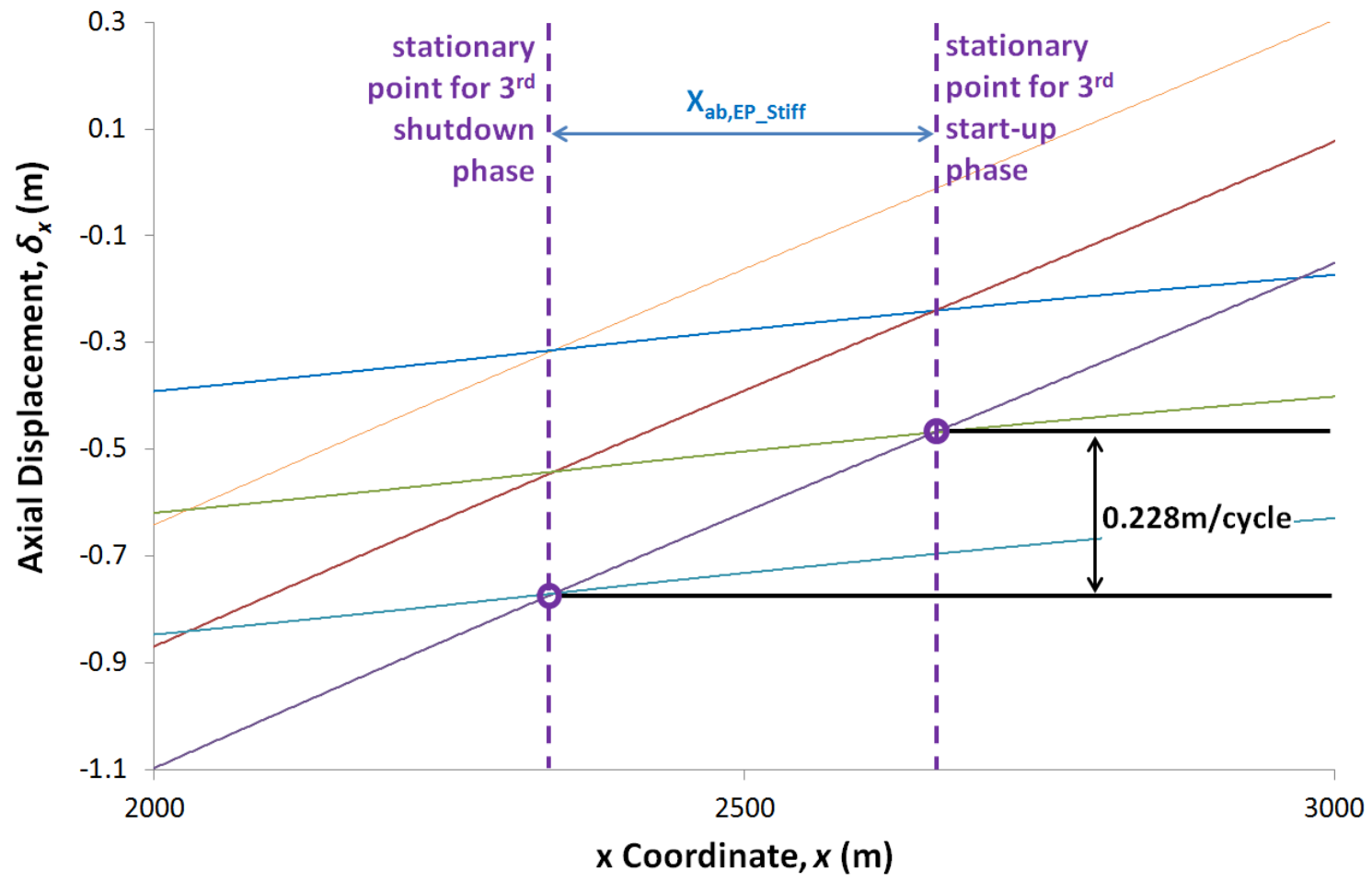

433

434 
Journal of Ocean Engineering

\section{Fig. $7 \delta_{x}$ plot for Soft Fit (Zoom)}

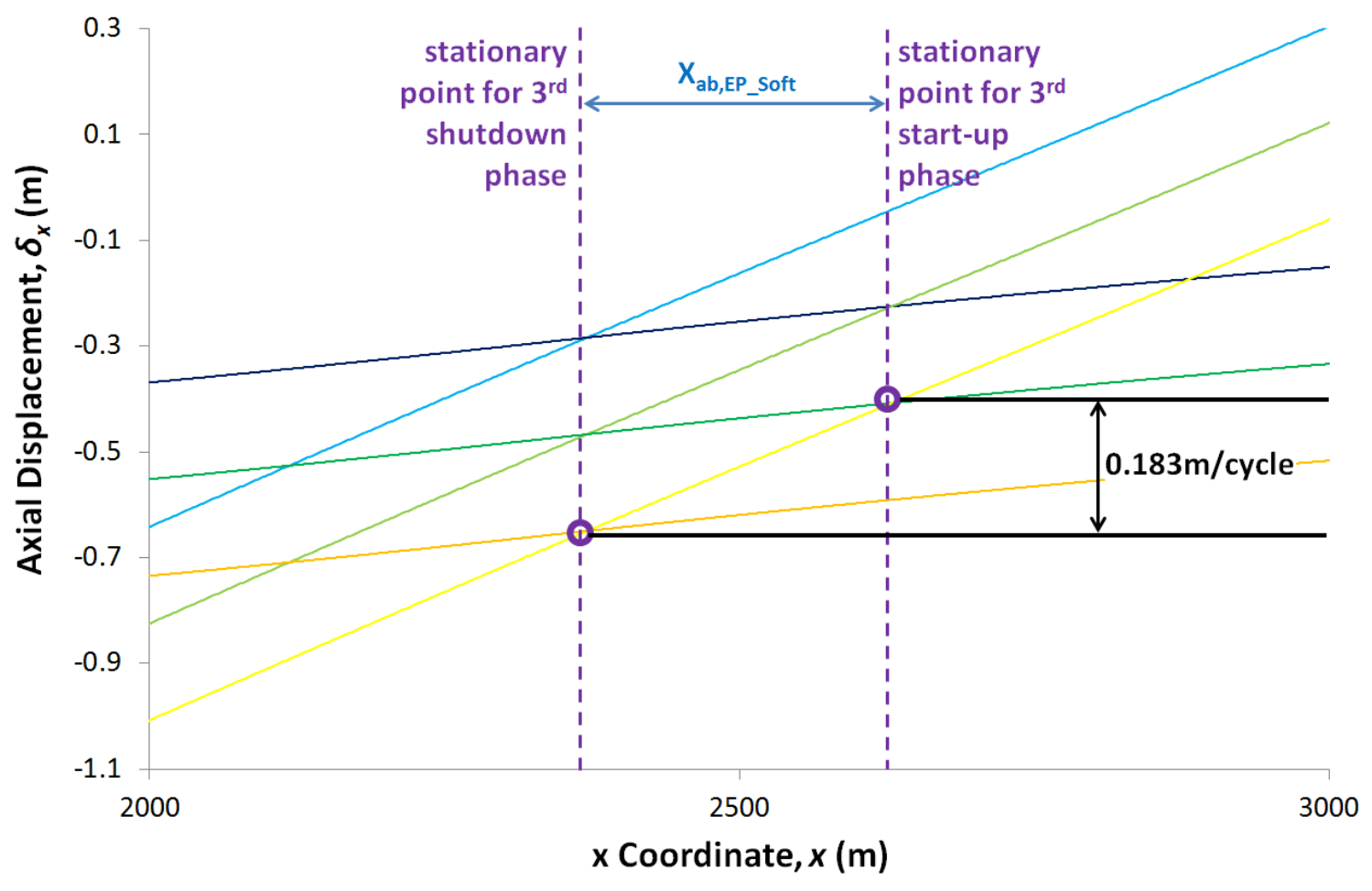

436
437

438 
Journal of Ocean Engineering

439

Fig. $8 \times$ Coordinate for the SPs
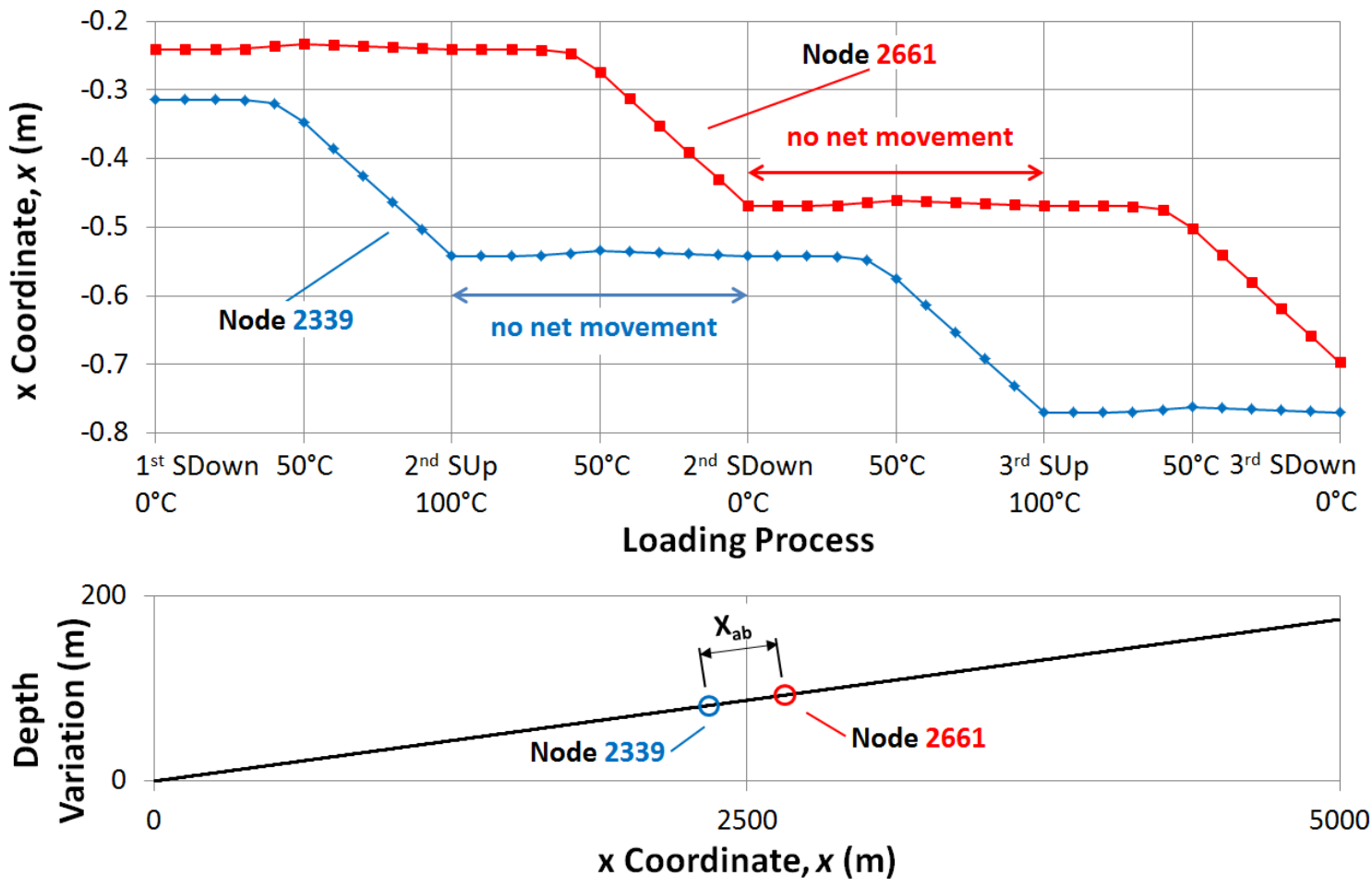

441

442 
Journal of Ocean Engineering

443 Fig. $9 X_{a b E P}$ results against $\delta_{m o b}$

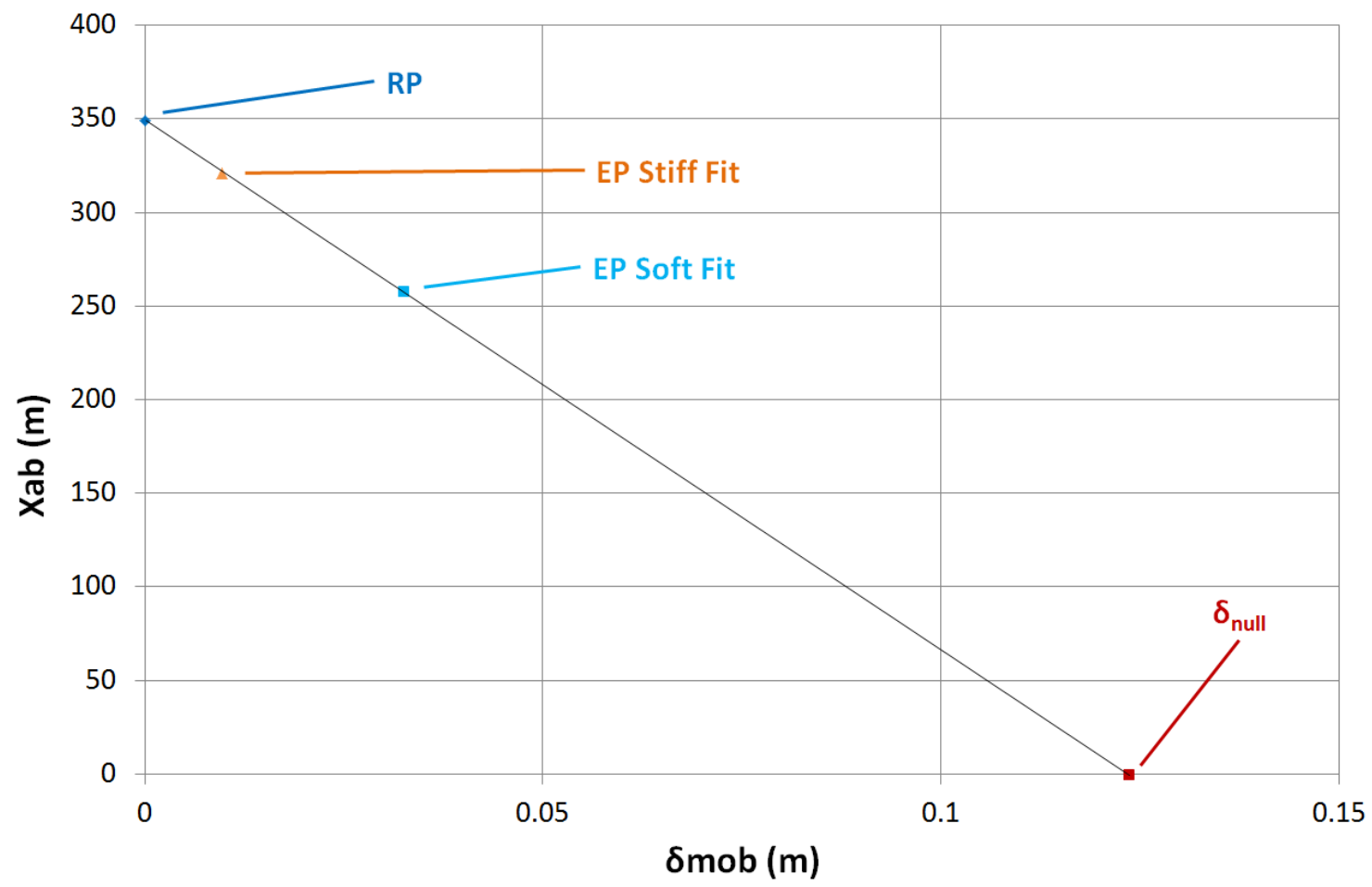

445

446 
Journal of Ocean Engineering

447 Fig. 10 Schematic plot accounting physical boundaries

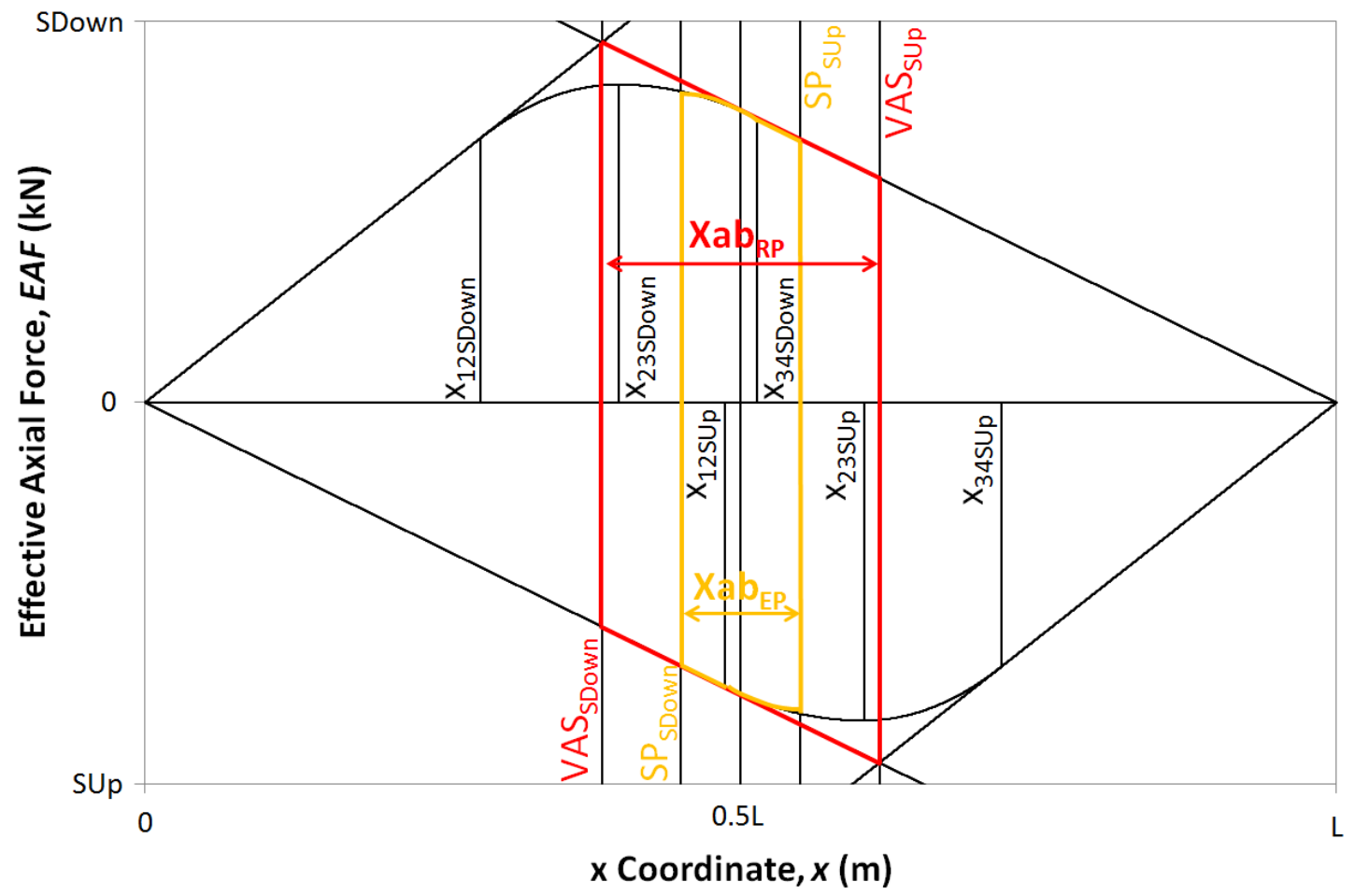

448
449

450 
Journal of Ocean Engineering

451 Fig. 11 Schematic EAF plot with the partial areas highlight

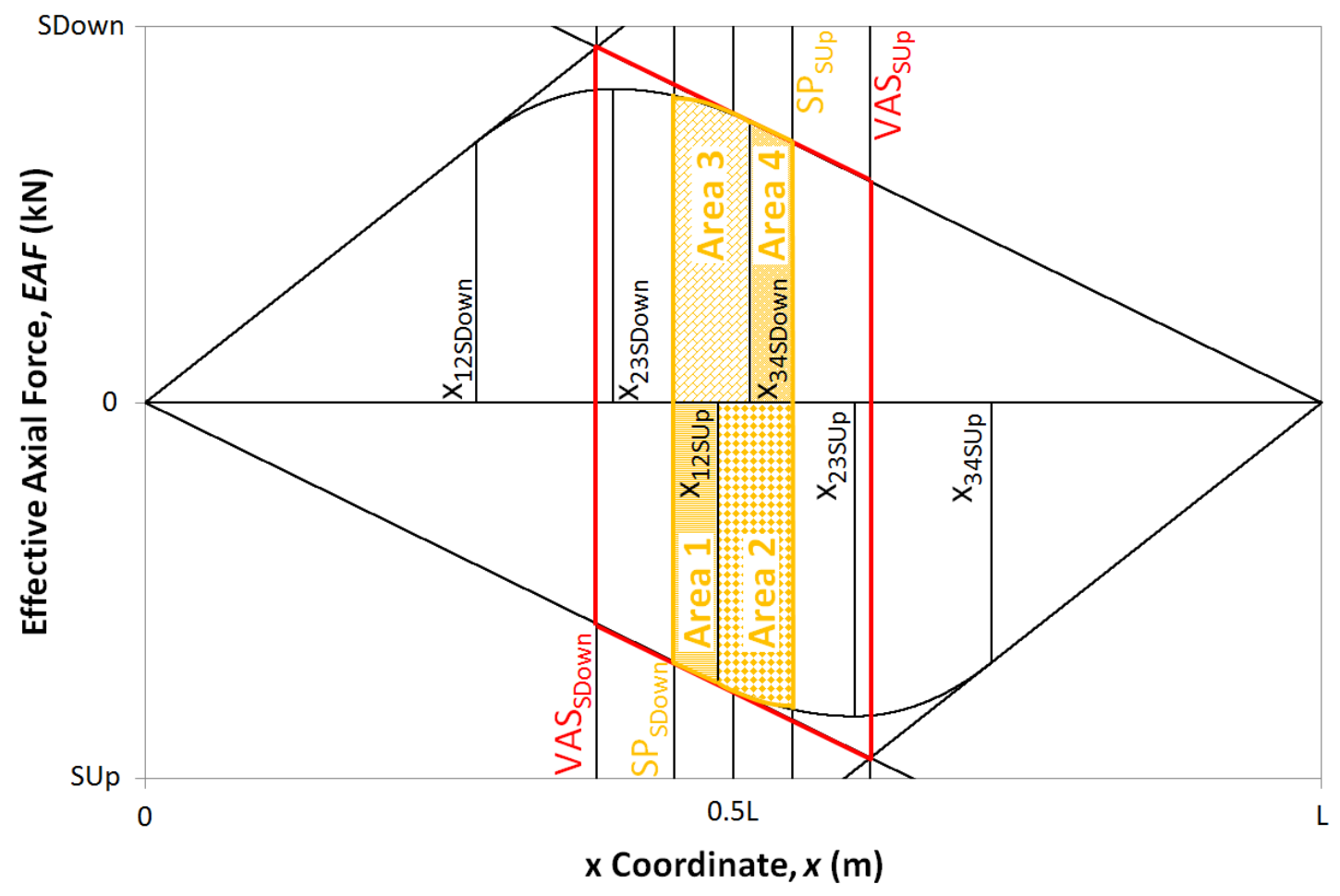

453

454 
Journal of Ocean Engineering

455

Fig. $12 X_{a b E P}$ results for $1^{\circ}$ slope

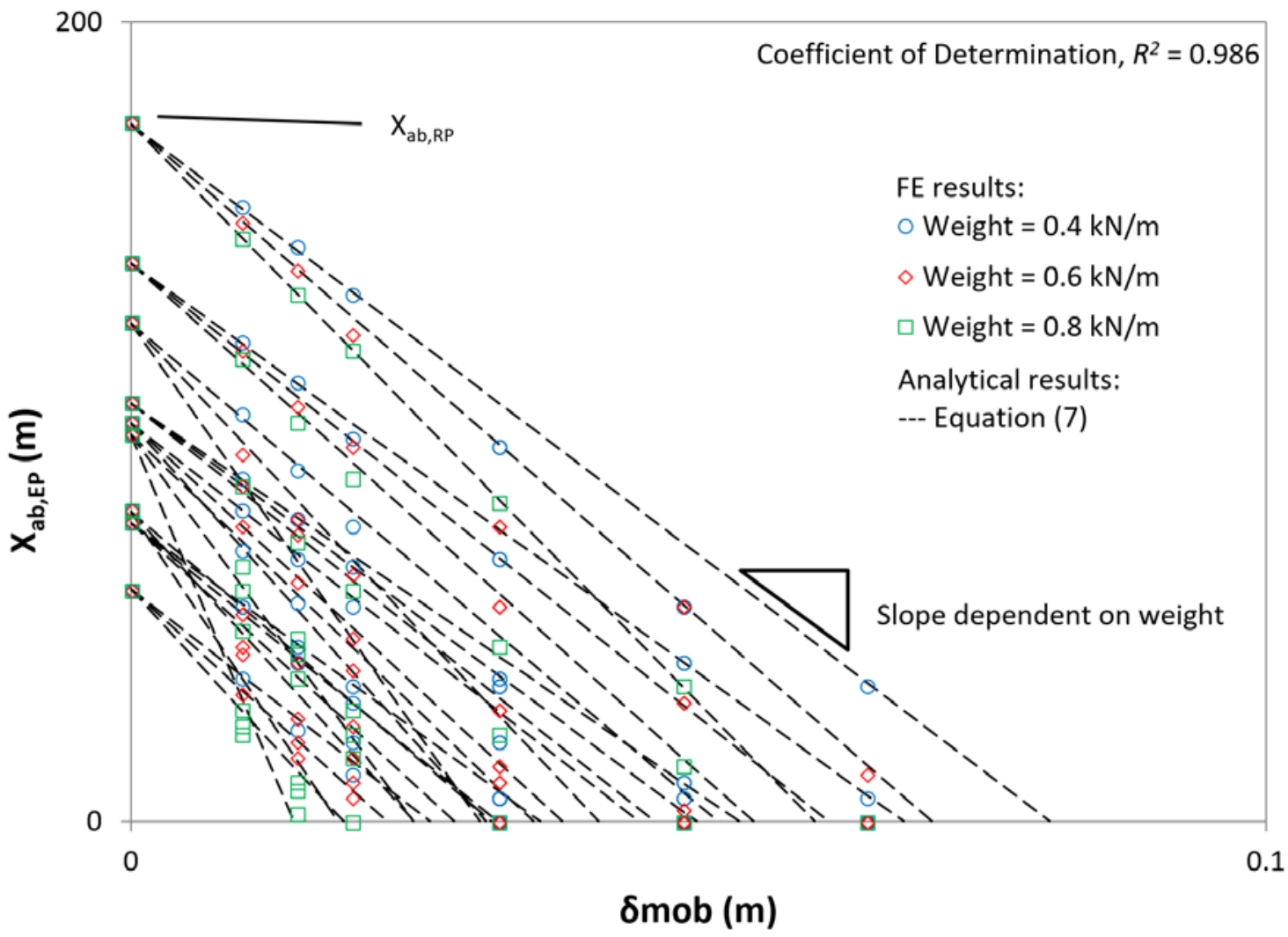

$\delta m o b(m)$ 
Journal of Ocean Engineering

458 Fig. $13 X_{a b E P}$ results - Numerical (FEA) \& Calculated (Equations) - for $1^{\circ}$ slope

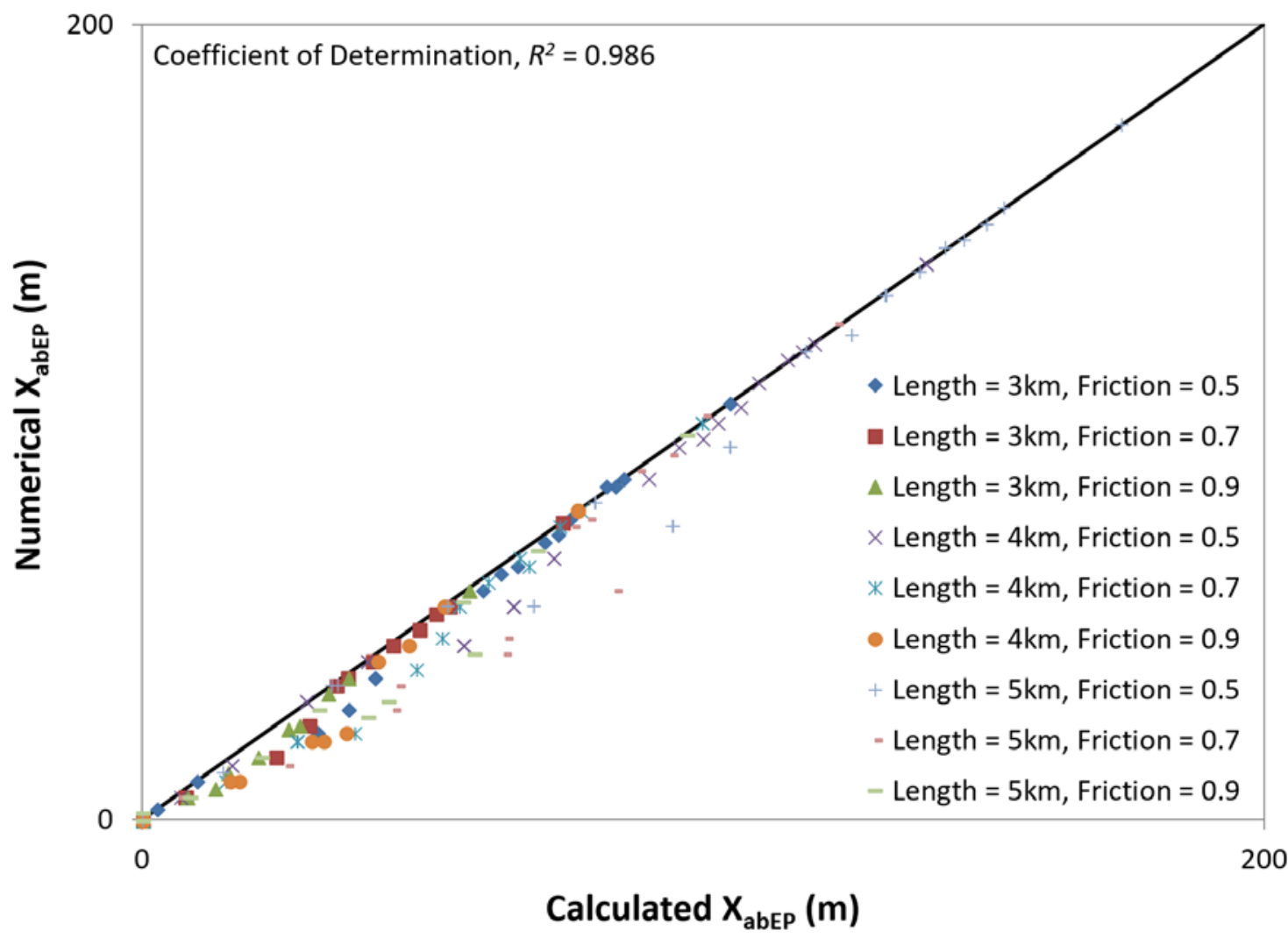


Journal of Ocean Engineering

461

Fig. $14 X_{a b E P}$ results for $2^{\circ}$ slope

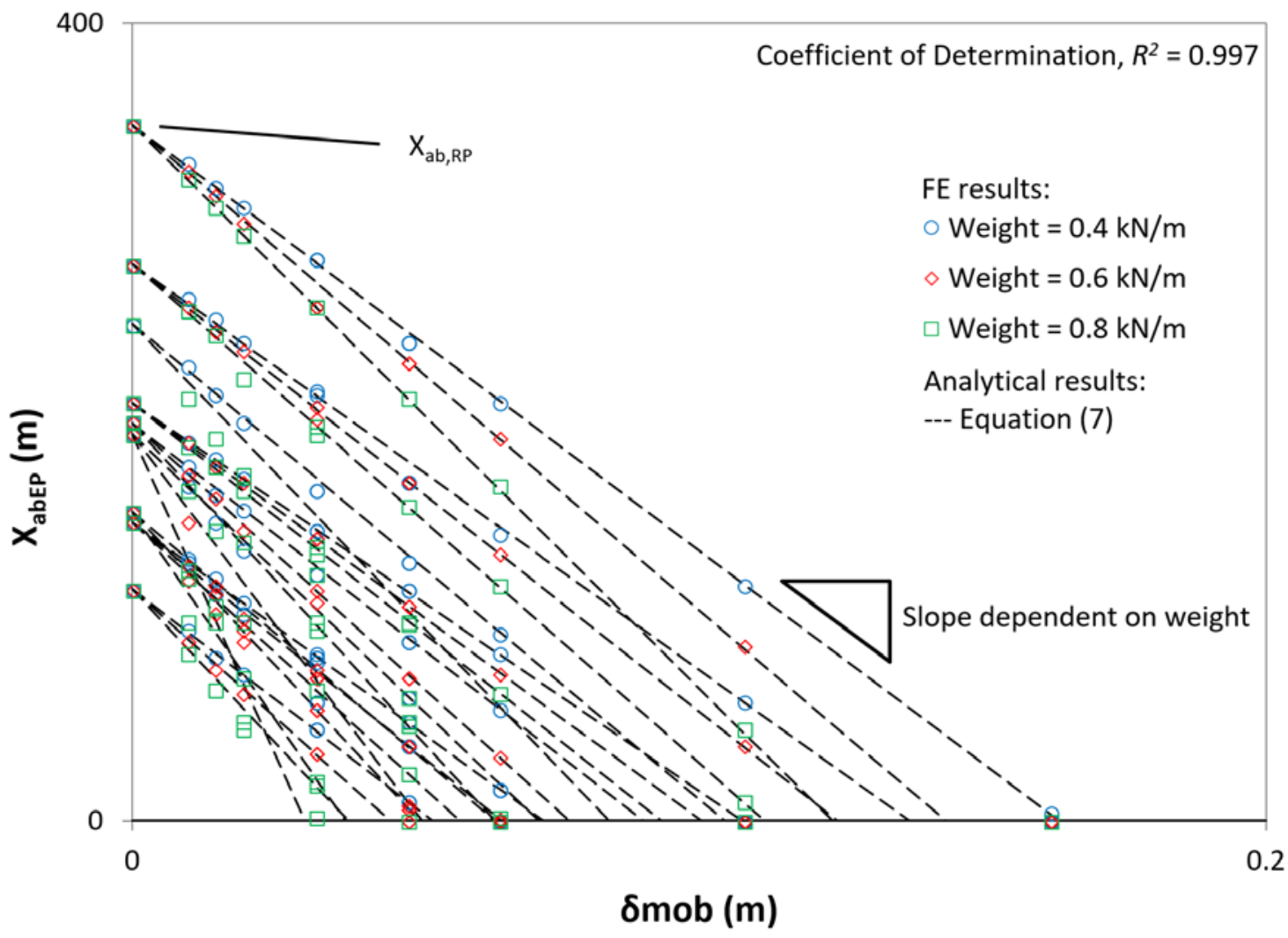

462

463

464 
Fig. $15 X_{a b E P}$ results - Numerical (FEA) \& Calculated (Equations) - for $2^{\circ}$ slope

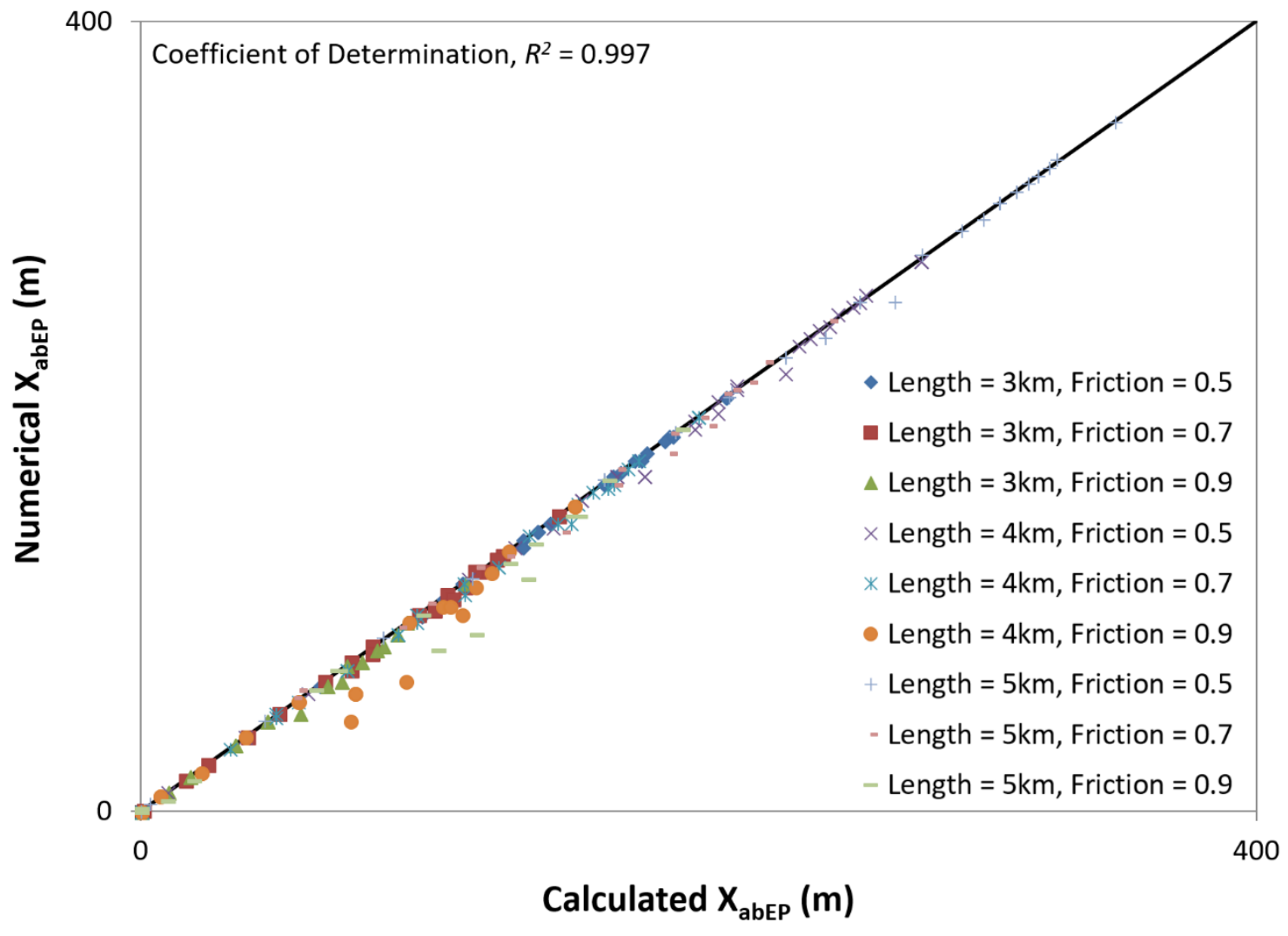


Journal of Ocean Engineering

Fig. $16 X_{a b E P}$ results for $3^{\circ}$ slope

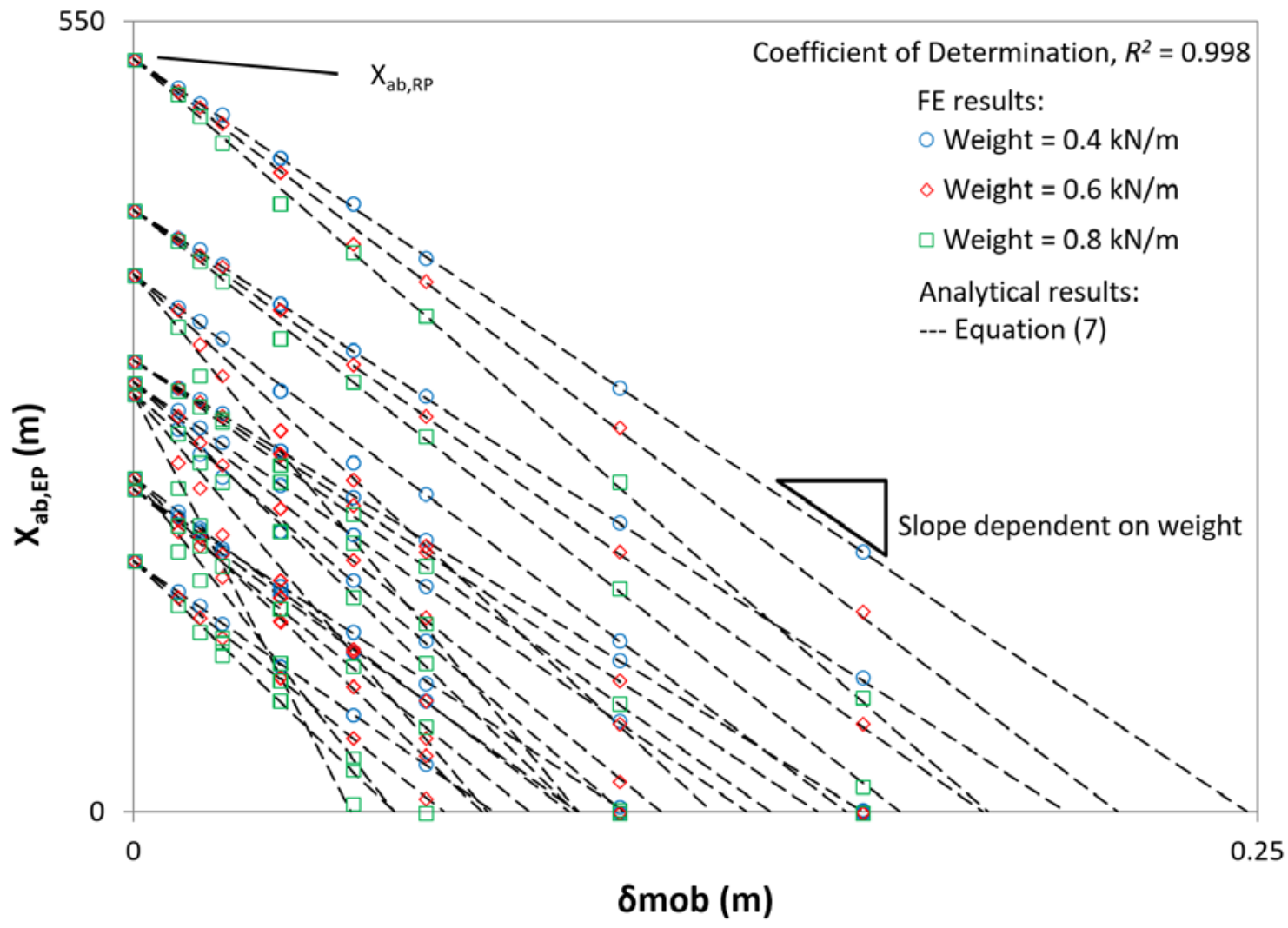

470

471

472 
Journal of Ocean Engineering

473 Fig. $17 X_{a b E P}$ results - Numerical (FEA) \& Calculated (Equations) - for $3^{\circ}$ slope

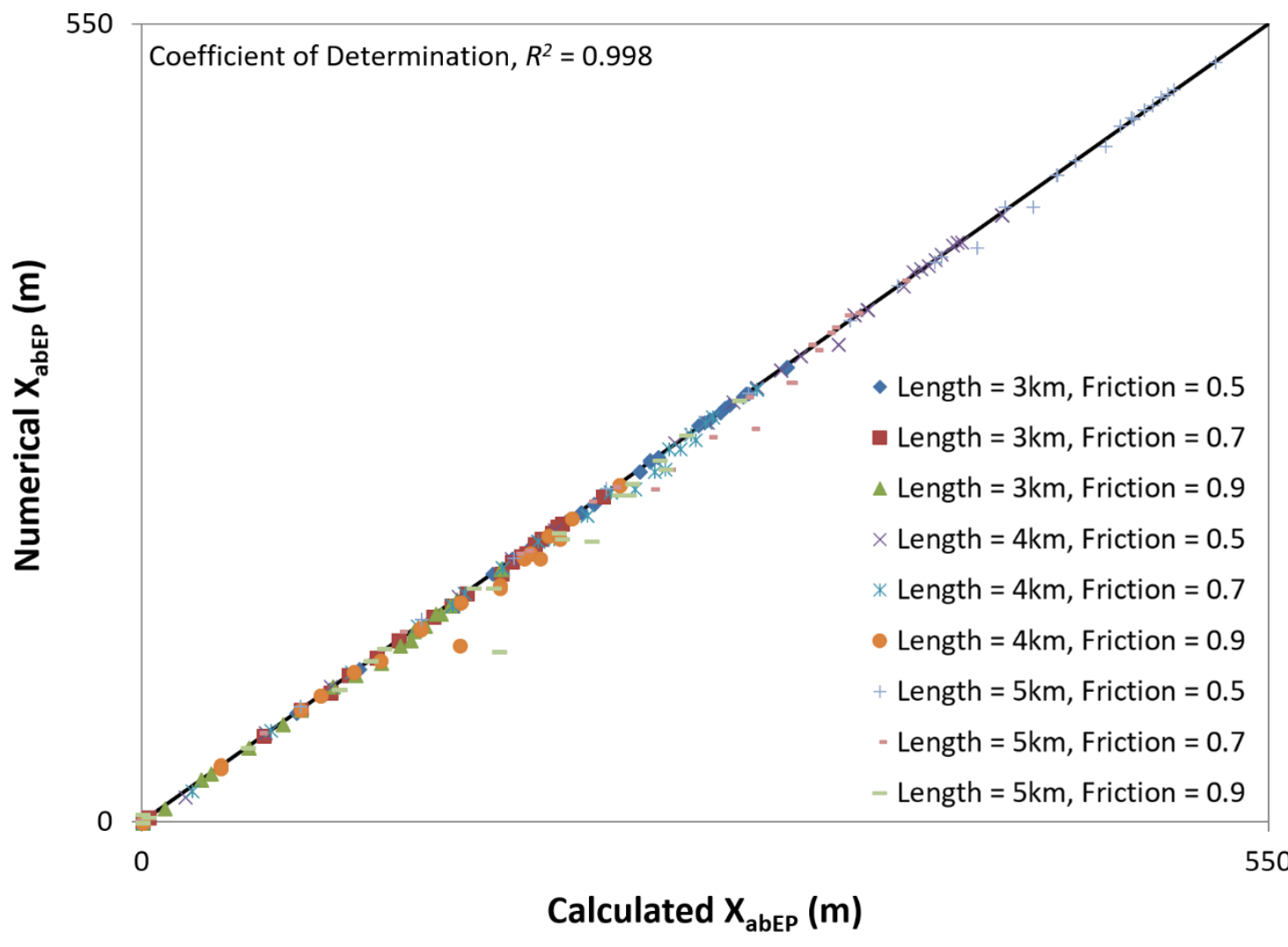

475

476 
Journal of Ocean Engineering

477 Fig. $18 W R_{E P}$ results for $1^{\circ}$ slope

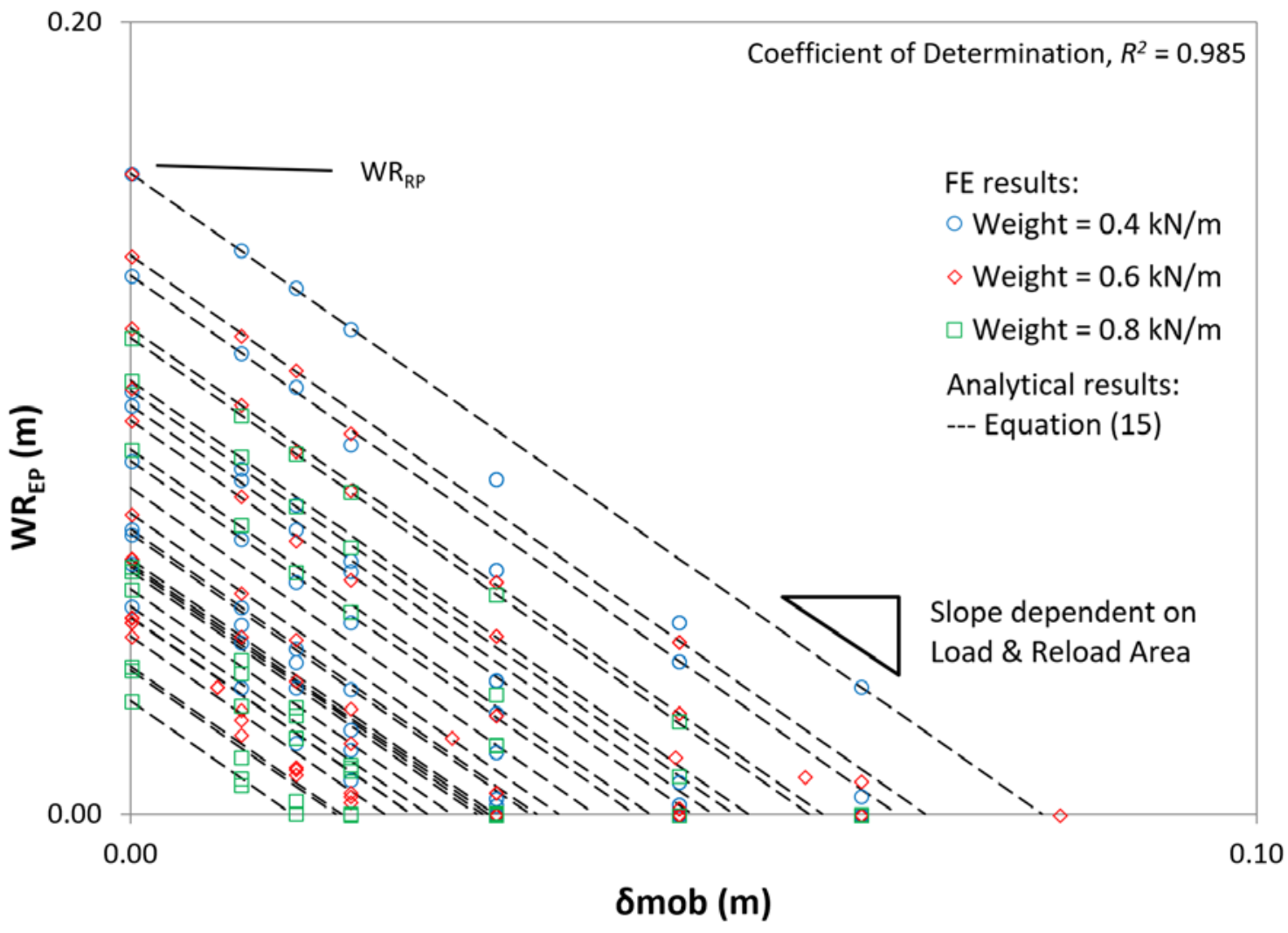

478

479

480 
481 Fig. $19 W R_{E P}$ results - Numerical (FEA) \& Calculated (Equations) - for $1^{\circ}$ slope

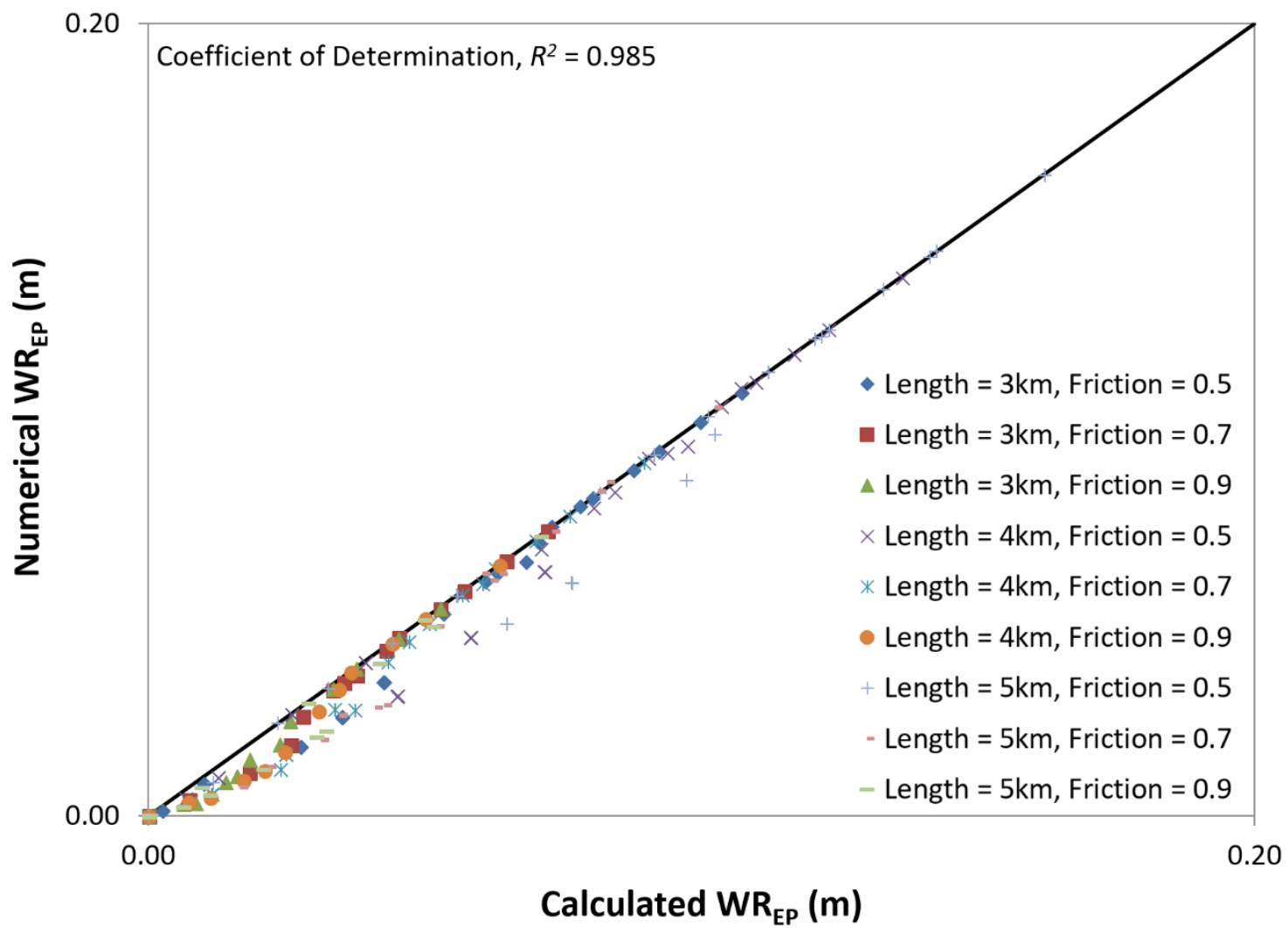


Journal of Ocean Engineering

485

Fig. $20 W R_{E P}$ results for $2^{\circ}$ slope

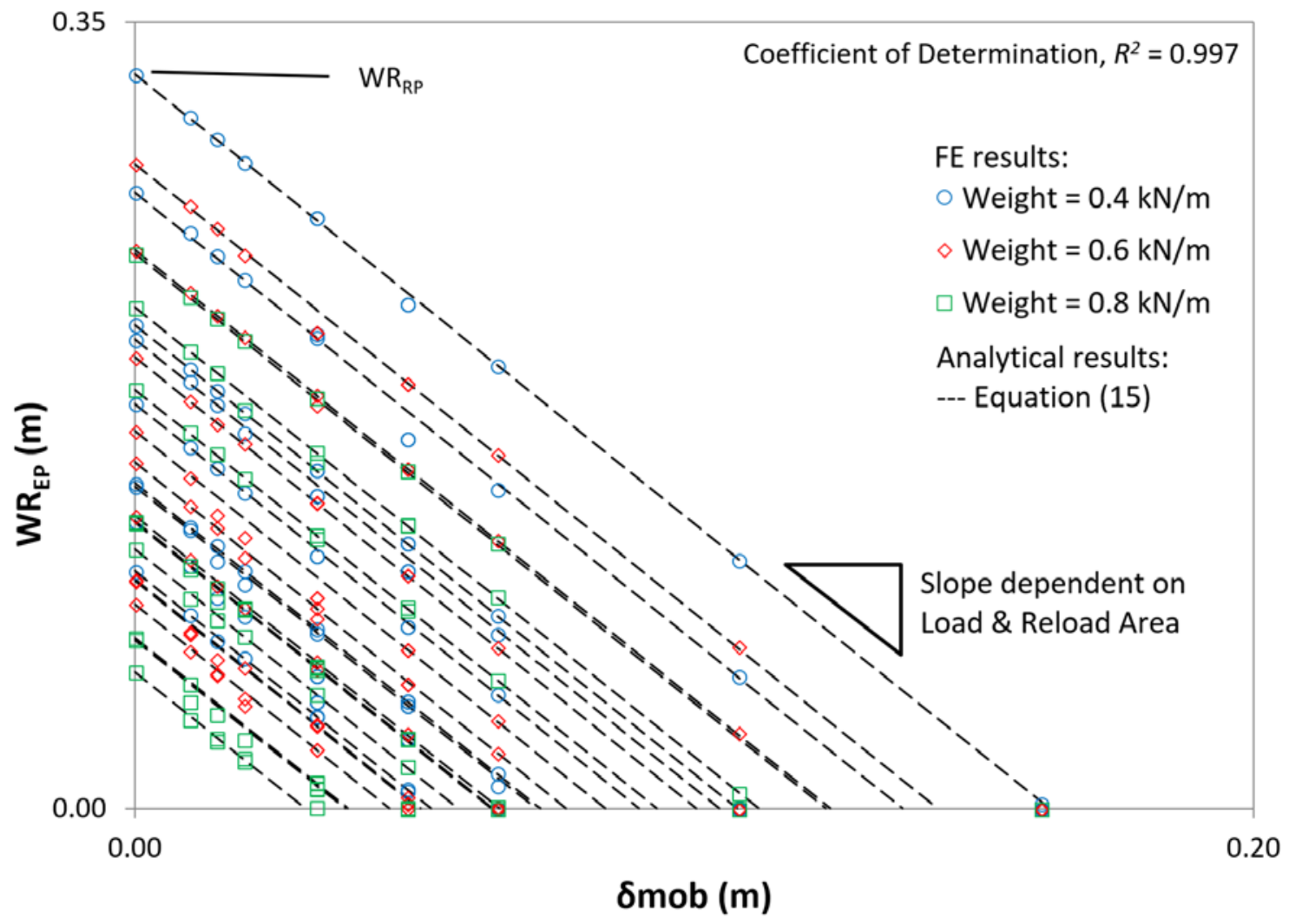

486

487

488 
Fig. $21 W R_{E P}$ results - Numerical (FEA) \& Calculated (Equations) - for $2^{\circ}$ slope

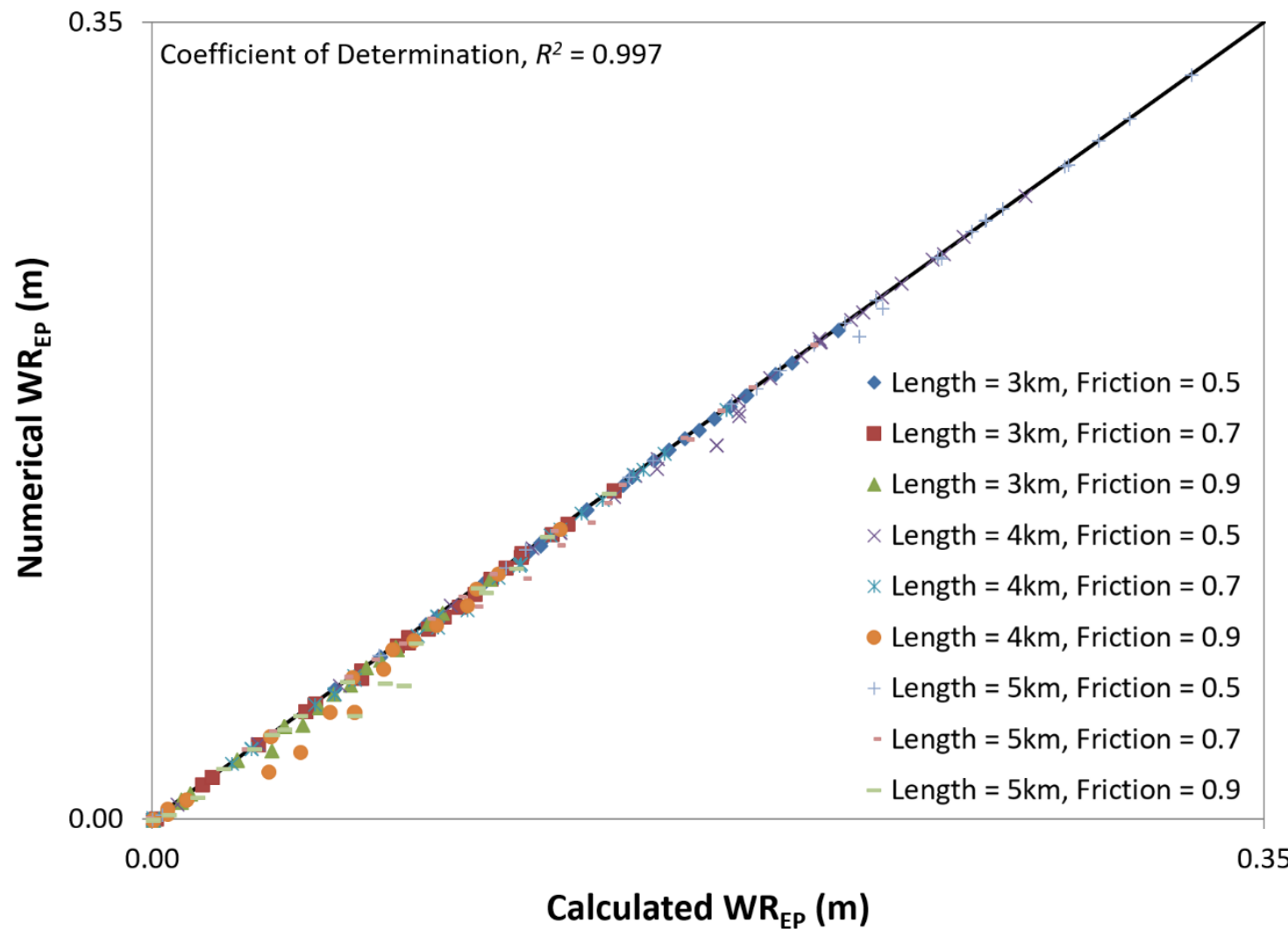


Journal of Ocean Engineering

493

Fig. $22 W R_{E P}$ results for $3^{\circ}$ slope

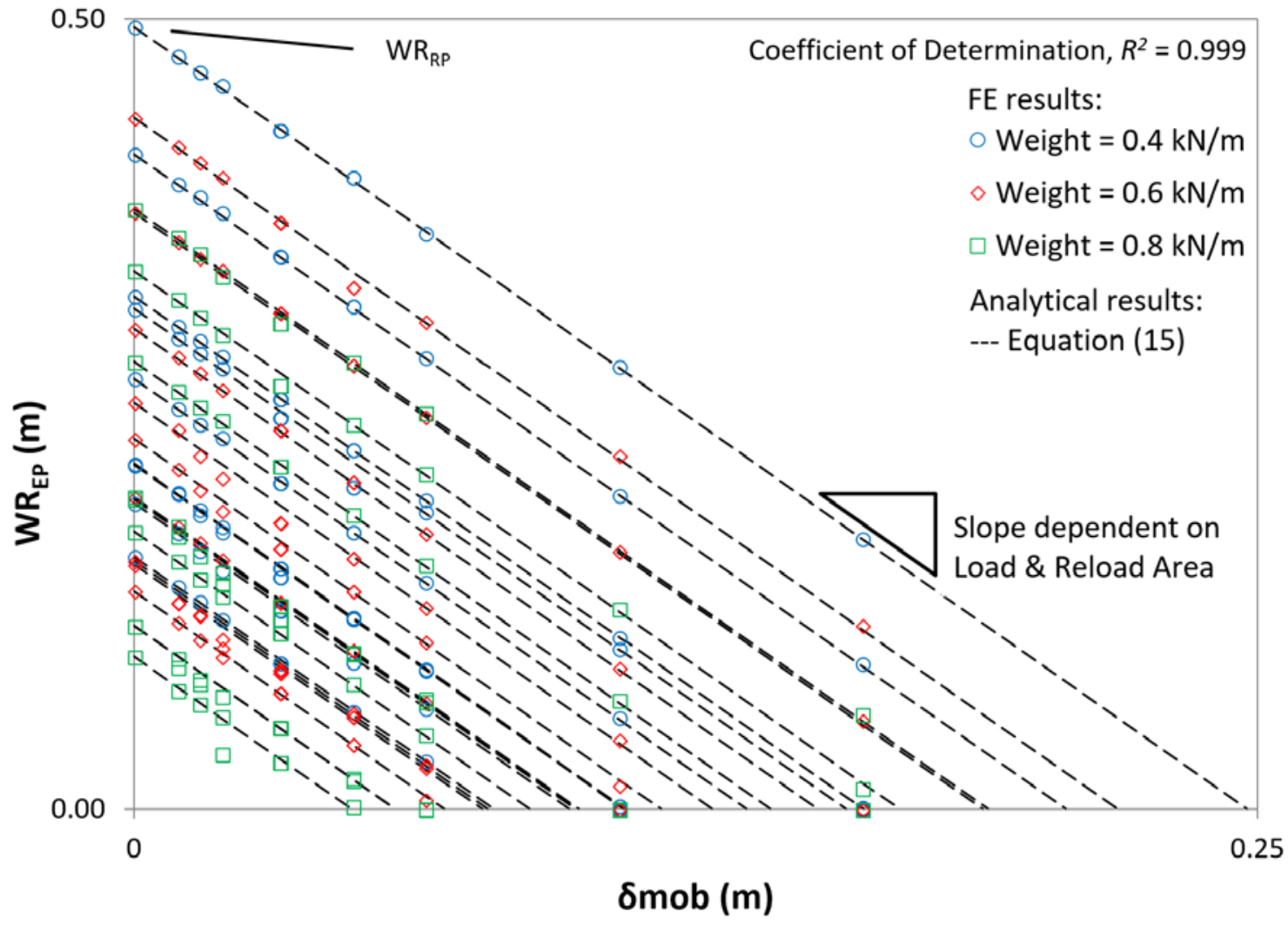

495

496 
Fig. $23 W R_{E P}$ results - Numerical (FEA) \& Calculated (Equations) - for $3^{\circ}$ slope

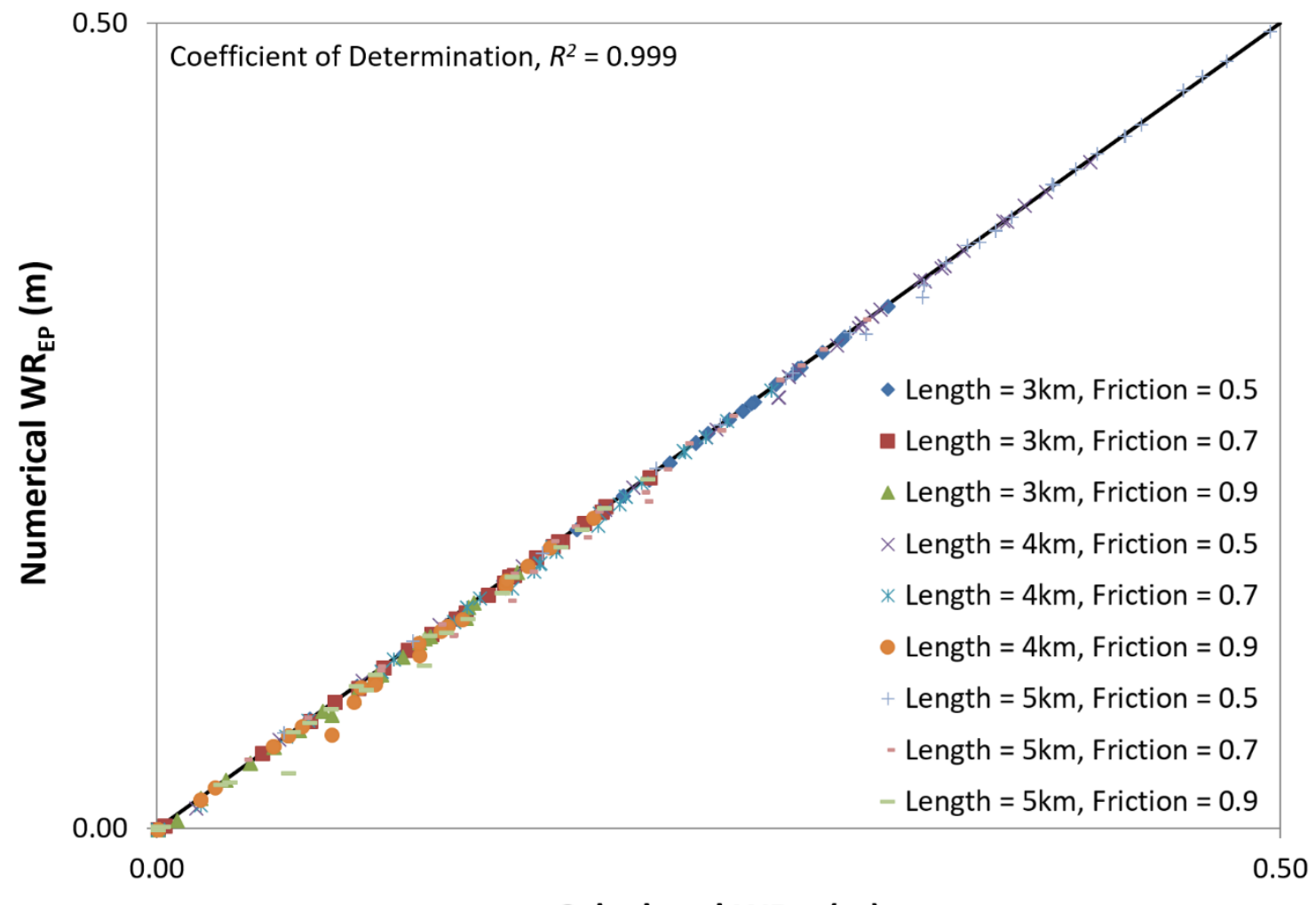

Calculated $W_{R_{E P}}(m)$ 
Journal of Ocean Engineering

501 TABLES

502 Table 1 Preliminary example properties

\begin{tabular}{ll} 
Parameter & Value \\
\hline Steel Outside Diameter, $O D$ & $0.3239 \mathrm{~m}$ \\
Steel Wall Thickness, $t$ & $0.0206 \mathrm{~m}$ \\
Length, $L$ & $5000 \mathrm{~m}$ \\
Seabed Slope, $B$ & $2.0^{\circ}$ \\
Temperature Variation, $\Delta T$ & $100^{\circ} \mathrm{C}$ \\
Pipe Submerged Weight, $W$ & $0.8 \mathrm{kN} / \mathrm{m}$ \\
Friction factor, $\mu$ & 0.5 \\
Steel Young's Modulus, $E$ & $2.07 \times 10^{11} \mathrm{~Pa}$ \\
Steel Poisson Coefficient, $v$ & 0.3 \\
Steel Thermal Expansion Coefficient, $\alpha$ & $1.165 \times 10^{-5 \circ} \mathrm{C}^{-1}$ \\
Mobilisation Distance for Stiff Fit, $\delta_{\text {mobstiff }}$ & $0.030 \mathrm{D}$ \\
Mobilisation Distance for Soft Fit, $\delta_{\text {mobsoft }}$ & $0.100 \mathrm{D}$
\end{tabular}

503

504 
Journal of Ocean Engineering

505 Table 2 RP analytical results

\begin{tabular}{ll} 
Parameter & Value \\
\hline$X_{a b, R P}$ & $349.208 \mathrm{~m}$ \\
$\Delta S_{S, R P}$ & $-1859.184 \mathrm{kN}$ \\
$W R_{R P}$ & $0.247 \mathrm{~m} /$ cycle
\end{tabular}

506

507 
Journal of Ocean Engineering

508 Table 3 EP FEA results

\begin{tabular}{lll} 
Parameter & Source & Value \\
\hline$X_{a b, R P}$ & EAF $\& \delta_{x}-$ Fig. 1, Fig. 2 and equation (1) & $349 \mathrm{~m}$ \\
$X_{a b, E P_{-} S t i f f}$ & EAF - Fig. 5 & $347 \mathrm{~m}$ \\
$X_{a b, E P_{-} \text {Stiff }}$ & $\delta_{X}-$ Fig. 6 & $321 \mathrm{~m}$ \\
$X_{a b, E P_{-} S o f t}$ & EAF - Fig. 5 & $343 \mathrm{~m}$ \\
$X_{a b, E P_{-} S o f t}$ & $\delta_{X}-$ Fig. 7 & $258 \mathrm{~m}$
\end{tabular}

509

510 
Journal of Ocean Engineering

511 Table 4 Pipeline zoning

\begin{tabular}{lll}
\hline Zone & Initial KP & Final KP \\
\hline$Z 1$ & 0 & $x_{12}$ \\
$Z 2$ & $x_{12}$ & $x_{23}$ \\
$Z 3$ & $x_{23}$ & $x_{34}$ \\
$Z 4$ & $x_{34}$ & $\mathrm{~L}$
\end{tabular}

512

513 
Journal of Ocean Engineering

514 Table 5 EAF boundary conditions

\begin{tabular}{|c|c|c|}
\hline$x$ coordinate & EAF & $\frac{d E A F}{d x}$ \\
\hline 0 & 0 & $W(\mu \cos \beta+\sin \beta)$ \\
\hline$x_{12}$ & $x_{12}[W(\mu \cos \beta+\sin \beta)]$ & $W(\mu \cos \beta+\sin \beta)$ \\
\hline$x_{23}$ & $?$ & 0 \\
\hline$x_{34}$ & $x_{34}[W(\mu \cos \beta-\sin \beta)]$ & $W(\mu \cos \beta-\sin \beta)$ \\
\hline $\mathrm{L}$ & 0 & $W(\mu \cos \beta-\sin \beta)$ \\
\hline
\end{tabular}

515

516 
Journal of Ocean Engineering

517 Table 6 FEA parametric variables

\begin{tabular}{llll} 
Parameter & Value A & Value B & Value C \\
\hline Length $(\mathrm{m})$ & 3000 & 4000 & 5000 \\
Weight $(\mathrm{kN} / \mathrm{m})$ & 0.4 & 0.6 & 0.8 \\
Friction $(-)$ & 0.5 & 0.7 & 0.9 \\
Slope $\left(^{\circ}\right)$ & 1 & 2 & 3 \\
\hline
\end{tabular}

518

519 
Journal of Ocean Engineering

\section{APPENDIX A}

521 In this appendix some auxiliary equations are listed, in order to keep the main

522 text concise, focused and direct.

523 From equation (19) to (22) the authors presented some secondary equations

524 related to item 4.

$$
\begin{gathered}
X_{a b, R P}=\left(V A S_{S U p}-V A S_{S D o w n}\right) \\
V A S_{S U p, R P}=\left(\frac{L+X_{a b, R P}}{2}\right) \\
V A S_{S D o w n, R P}=\left(\frac{L-X_{a b, R P}}{2}\right) \\
\Delta P=-\left(p_{2}-p_{1}\right) A_{i}(1-2 v)-E A_{s} \alpha\left(T_{2}-T_{1}\right)
\end{gathered}
$$

525

526 


\section{APPENDIX B}

529 accordingly with [12].

530 a Basic Mechanics Revision

$$
\begin{gathered}
\varepsilon_{\text {Mech }}=\frac{F}{E A} \\
\varepsilon_{\text {Total }}=\frac{d \delta}{d x} \\
\left(\varepsilon_{\text {Mechanical }}+\varepsilon_{\text {Thermal }}\right)=\frac{d \delta}{d x} \\
\frac{d^{2} \delta}{d x^{2}}=\frac{1}{E A} \frac{d F}{d x}+\alpha \Delta T
\end{gathered}
$$

531 b Longitudinal Coordinate

532 The longitudinal coordinate, in equation (8) referred to as $x$, was substituted by

533 the section distance, $s$, as expressed by equation (27); where $x$ is the absolute KP value

534 of the section in question and $x_{23}$ is the boundary KP value for the case assessed, as

535 previously explained.

$536 \quad c$ Factor $\xi$

$$
s=\left|x-x_{23}\right|
$$

537 To get this factor expression, put all zones apart and do the calculations only for

$538 Z 1$, the other zones will be later checked to prove whether this result is valid or not. Hence, putting together equations (8) and (11) we can achieve the following

540 system of equations:

$$
\left\{\begin{array}{c}
\delta=K_{1} e^{\xi_{Z 1} * S}+K_{2} e^{-\xi_{Z 1} * S} \\
\frac{d F}{d x}=\left(\frac{\mu W_{Z 1}}{\delta_{m o b}}\right) \delta \\
\frac{d F}{d x}=E A\left(K_{1} \xi_{Z 1}{ }^{2} e^{\xi_{Z 1} * S}+K_{2} \xi_{Z 1}{ }^{2} e^{-\xi_{Z 1} * s}\right)
\end{array}\right.
$$

And from this system of equations, we can extract: 
Journal of Ocean Engineering

$$
\xi_{Z 1}=\sqrt{\left(\frac{\mu W_{Z 1}}{E A \delta_{m o b}}\right)}
$$

From the final shape of its expression, we can conclude that for zones $Z 1$ and $Z 2$,

$543 \xi$ has the same value; and, this is also valid for zones $Z 3$ and $Z 4$. Then, there are actually

544 only two values for factor $\xi_{,} \xi_{z 1}$ and $\xi_{z 4}$ applicable for zones $Z 1$ \& Z2 and Z3 \& Z4,

545 respectively.

546 d Constants $K_{1}$ and $K_{2}$

547 Analogously to Randolph's equations (4.27) and (4.28), we needed to define a

548 pair of equations suited to the present problem, to be considered at a single position of

549 the pipe.

550 Equation (30) is related to $x_{23}$ displacement, while equation (31) is related to its

551 third derivative through the second derivative of force.

$$
\begin{gathered}
\delta_{(x 23)}=\left(K_{1} e^{\xi_{Z 1} * S}+K_{2} e^{-\xi_{Z 1} * S}\right)_{\left(x_{23}\right)} \\
\frac{d^{2} F}{d x^{2}}{ }_{(x 23)}=E A \xi_{Z 1}{ }^{3}\left(K_{1} e^{\xi_{Z 1} * S}-K_{2} e^{-\xi_{Z 1} * S}\right)_{\left(x_{23}\right)}
\end{gathered}
$$

552 For $x_{23}$, we can simplify the exponential portions as equal to 1 , because the $s$

553 exponent will assume the value of 0 (zero). The notation $Z 1$ was used in this item, but it

554 could be used $Z 4$, as well, because $x_{23}$ is the limit between the different zones.

555 Therefore, because of point $x_{23}$ 's nature, equation (30) and equation (31) might be

556 rewritten with $Z 4$ indexes. This also means that the force acting at $x_{23}$ might be

557 dependent on $Z 1$ or $Z 4$ and they must provide the same force result.

558 Tackling first equation (30), we will have - analogously to Randolph's equation

$559(4.28)-$ using the $\delta_{x}$ boundary conditions (item 8i): 
Journal of Ocean Engineering

$$
\begin{gathered}
\delta_{\left(x_{23}\right)}=0 \\
\left(K_{1} e^{\xi_{Z 1} * S}+K_{2} e^{-\xi_{Z 1} * S}\right)_{\left(x_{23}\right)}=0 \\
\left(K_{1}+K_{2}\right)_{\left(x_{23}\right)}=0
\end{gathered}
$$

560

Before handling equation (31), we need to take a step back and look at the

561 following relations:

$$
\begin{gathered}
\frac{d^{2} F}{d x^{2}}=\frac{d \frac{d F}{d x}}{d x} \\
\frac{d^{2} F}{d x^{2}}=\frac{d\left(\frac{\mu W_{Z 1}}{\delta_{m o b}}\right) \delta}{d x} \\
\frac{d^{2} F}{d x^{2}}=\left(\frac{\mu W_{Z 1}}{\delta_{m o b}}\right) \frac{d \delta}{d x} \\
\frac{d^{2} F}{d x^{2}}=\left(\frac{\mu W_{Z 1}}{\delta_{\text {mob }}}\right) \varepsilon_{\text {Total }} \\
\frac{d^{2} F}{d x^{2}}=\left(\frac{\mu W_{Z 1}}{\delta_{m o b}}\right)\left(\varepsilon_{\text {Mechanical }}+\varepsilon_{\text {Thermal }}\right) \\
\left.\frac{\mu W_{Z 1}}{\delta_{\text {mob }}}\right)\left(\frac{F}{E A}+\alpha \Delta T\right)=\frac{\mu W_{Z 1}}{E A \delta_{\text {mob }}} * F+\frac{\mu W_{Z 1} \alpha \Delta T}{\delta_{m o b}}
\end{gathered}
$$

563 have:

$$
\left(K_{1}-K_{2}\right)_{\left(x_{23}\right)}=\left(\frac{\mu W_{Z 1}}{\xi_{Z 1}{ }^{3} E A^{2} \delta_{m o b}} * F+\frac{\mu W_{Z 1} \alpha \Delta T}{\xi_{Z 1}{ }^{3} E A \delta_{m o b}}\right)_{\left(x_{23}\right)}
$$

564 Working with equations (32) and (33) as a system, we'll achieve:

$$
\begin{aligned}
K_{1_{\left(x_{23}\right)}} & =\left(\frac{\mu W_{Z 1}}{2 \xi_{Z 1}{ }^{3} E A \delta_{m o b}}\left[\frac{1}{E A} * F+\alpha \Delta T\right]\right)_{\left(x_{23}\right)} \\
K_{2\left(x_{23}\right)} & =-\left(\frac{\mu W_{Z 1}}{2 \xi_{Z 1}{ }^{3} E A \delta_{m o b}}\left[\frac{1}{E A} * F+\alpha \Delta T\right]\right)_{\left(x_{23}\right)}
\end{aligned}
$$

$565 \quad$ Algebraically manipulating $\xi$ we can simplify equation (35) as: 


$$
\begin{gathered}
K_{1_{\left(x_{23}\right)}}=\left(\frac{1}{2} \sqrt{\frac{E A \delta_{m o b}}{\mu W_{Z 1}}}\left[\frac{1}{E A} * F+\alpha \Delta T\right]\right)_{\left(x_{23}\right)} \\
K_{2\left(x_{23}\right)}=-\left(\frac{1}{2} \sqrt{\frac{E A \delta_{m o b}}{\mu W_{Z 1}}}\left[\frac{1}{E A} * F+\alpha \Delta T\right]\right)_{\left(x_{23}\right)}
\end{gathered}
$$

Or if we prefer:

$$
\begin{aligned}
& K_{1\left(x_{23}\right)}=\left(\frac{1}{2} \sqrt{\frac{E A \delta_{m o b}}{\mu W_{Z 1}}} \varepsilon_{\text {Total }}\right)_{\left(x_{23}\right)} \\
& K_{2\left(x_{23}\right)}=-\left(\frac{1}{2} \sqrt{\frac{E A \delta_{m o b}}{\mu W_{Z 1}}} \varepsilon_{\text {Total }}\right)_{\left(x_{23}\right)}
\end{aligned}
$$

568 on the force acting at $x_{23}$. At this point, the value provided by Carr's solution is applied.

$569 \quad$ By the expressions shown in equation (36), it was foreseen that the impact of the

570 rigid-plastic force value would be extremely small, once the force is divided by the axial

571 stiffness. This prediction was later confirmed when the results were compared for $K_{1}$

572 and $K_{2}$ calculated with rigid-plastic and elastic-perfectly-plastic soils responses (the 573 difference was $0.003 \%)$. 